\title{
On the estimation of a monotone conditional variance in nonparametric regression
}

\author{
Holger Dette • Kay Pilz
}

Received: 17 August 2005 / Revised: 7 December 2006 / Published online: 5 April 2007

(C) The Institute of Statistical Mathematics, Tokyo 2007

\begin{abstract}
A monotone estimate of the conditional variance function in a heteroscedastic, nonparametric regression model is proposed. The method is based on the application of a kernel density estimate to an unconstrained estimate of the variance function and yields an estimate of the inverse variance function. The final monotone estimate of the variance function is obtained by an inversion of this function. The method is applicable to a broad class of nonparametric estimates of the conditional variance and particularly attractive to users of conventional kernel methods, because it does not require constrained optimization techniques. The approach is also illustrated by means of a simulation study.
\end{abstract}

Keywords Nonparametric regression - Heteroscedasticity - Variance function · Monotonicity · Order restricted inference

\section{Introduction}

In regression analysis the assumption of homoscedasticity is often not satisfied and the efficiency of the statistical analysis can be improved substantially by taking heteroscedasticity into account. The classical example is the weighted least squares method, which requires estimates of the variance function. Other examples, where the estimation of the conditional variance is important include the choice of a local bandwidth in nonparametric regression (see Müller and Stadtmüller 1987, Fan and Gijbels 1995), the construction of confidence intervals for the conditional expectation (see Carroll 1987, Fan and Gijbels 1996) and quality control (see Box 1988). In contrast to the problem of estimating

H. Dette (更) K. Pilz

Fakultät für Mathematik, Ruhr-Universität Bochum, 44780 Bochum, Germany

e-mail: holger.dette@ruhr-uni-bochum.de 
the conditional mean much less effort has been spent on the construction of nonparametric variance function estimators. Carroll (1982) developed kernel estimators in the context of linear regression, Müller and Stadtmüller (1987) and Hall and Carroll (1989) analyzed kernel-type estimators without assuming a parametric form of the mean function, and Müller and Stadtmüller (1993) studied a broad class of estimators of the conditional variance, which are representable as quadratic forms. Local polynomial variance function estimators have been proposed by Fan and Gijbels (1995) and Ruppert et al. (1997), where the latter authors also consider the problem of estimating derivatives of the variance function, a topic with applications in engineering. More recently the estimation of the conditional variance was considered by Fan and Yao (1998) in a time series context and by Yu and Jones (2004), who proposed a localized normal likelihood approach.

In many applications monotone estimates of the regression and variance function are required because of physical considerations. Such examples typically appear in growth curve models or in models, where the conditional variance is a function of the conditional mean, which depends monotonically on an explanatory variable. In contrast to the problem of estimating a monotone conditional expectation (see e.g. Brunk 1955, Mukerjee 1988, Mammen 1991, Hall and Huang 2001, among many others), the problem of estimating a monotone variance function has not been considered so far in the literature. In the present paper we propose a simple and efficient method for the estimation of a monotone conditional variance, which is based on the evaluation of a kernel density estimate from some (not necessarily monotone) estimated values of the variance function. This idea was introduced by Dette et al. (2006) in the context of estimating a monotone regression function and will be adapted to the specific problem of statistical inference for the conditional variance. The method produces an estimate of the inverse of the monotone variance function and is applicable to any of the unconstrained variance function estimators mentioned in the previous paragraph. The main differences between the work of these authors and the present paper are the following. While Dette et al. (2006) considered the problem of estimating the regression function, the focus of our work is the estimation of the conditional variance. This introduces some additional dependencies, which change parts of the asymptotic analysis, because the original data has to be replaced by dependent (squared) nonparametric residuals. A further difference is the order of the smoothing parameters. Dette et al. (2006) mainly consider smoothing parameters in the density and regression step, which are of the same order, while in the present paper the smoothing parameter for the density estimate is assumed to be of smaller order than the smoothing parameter in the regression step.

In Sect. 2 we introduce some general notation and explain the basic idea of monotonizing a function by kernel density estimation. Because most work on unconstrained variance estimation suggests smoothing squared residuals from a nonparametric fit or pseudo-residuals by kernel smoothing we mainly restrict ourselves to this type of variance function estimators, but the results of the paper remain valid for other estimation methods. In Sect. 3 we prove 
asymptotic normality of the new estimate and show that it is first order asymptotically equivalent to the unconstrained variance function estimate. We also mention the corresponding statements for the local polynomial estimators of the conditional variance introduced by Fan and Gijbels (1995) and Ruppert et al. (1997). For the sake of brevity we restrict ourselves to the case of a nonparametric regression model with a fixed design and independent errors, but extensions to more general models (random design, time series) are briefly mentioned in Sect. 3.3. Finally, in Sect. 4 a small simulation study and a data example are presented which illustrate the finite sample properties of the new monotone variance function estimates. Finally some of the more technical arguments are deferred to the appendix in Sect. 5.

\section{Preliminaries: monotonizing by kernel density estimation}

Consider the common nonparametric regression model

$$
Y_{i, n}=m\left(x_{i, n}\right)+\sqrt{s\left(x_{i, n}\right)} \varepsilon_{i, n}
$$

where $0 \leq x_{1, n}<x_{2, n}<\cdots<x_{n, n}=1$ are fixed design points satisfying

$$
\int_{0}^{x_{i, n}} f(t) \mathrm{d} t=\frac{i}{n}, \quad i=1, \ldots, n
$$

for a strictly positive design density $f:[0,1] \rightarrow \mathbb{R}$ (see Sacks and Ylvisaker $1970), m:[0,1] \rightarrow \mathbb{R}$ denotes the regression and $s:[0,1] \rightarrow \mathbb{R}$ is a positive variance function. The errors $\varepsilon_{1, n}, \ldots, \varepsilon_{n, n}$ are assumed to be independent identically distributed with mean $E\left[\varepsilon_{i, n}\right]=0$, variance $\operatorname{Var}\left(\varepsilon_{i, n}\right)=1$ and fourth moment $E\left[\varepsilon_{i, n}^{4}\right]=m_{4}\left(x_{i, n}\right)$, where $m_{4}:[0,1] \rightarrow \mathbb{R}^{+}$is a smooth function. For the sake of a simple notation we omit the index $n$, whenever it is clear from the context, i.e. we use the notation $Y_{i}, x_{i}, \varepsilon_{i}$ instead of $Y_{i, n}, x_{i, n}, \varepsilon_{i, n}$ in such cases. We assume that the design density $f$ and the variance function $s$ are two times continuously differentiable and that the regression function $m$ satisfies certain smoothness conditions which will be specified below. Moreover, the variance function is assumed to be strictly monotone and we are interested in a nonparametric estimate of this function, which also satisfies this restriction.

In order to fix ideas let $s$ denote an arbitrary strictly increasing function on the interval $[0,1]$, then the inverse of $s$ can be represented as

$$
s^{-1}(t)=\int_{0}^{1} I\{s(x) \leq t\} \mathrm{d} x .
$$


Note that this function is not necessarily smooth, but smoothing can easily be accomplished by considering the function

$$
s^{-1}\left(t, h_{d}\right)=\frac{1}{h_{d}} \int_{0}^{1} \int_{-\infty}^{t} K_{d}\left(\frac{s(x)-u}{h_{d}}\right) \mathrm{d} u \mathrm{~d} x,
$$

where $h_{d}$ is a bandwidth satisfying $h_{d} \rightarrow 0$ with increasing sample size and $K_{d}$ is a two times continuously differentiable, symmetric kernel with compact support, say $[-1,1]$. Note that for $h_{d} \rightarrow 0$ we have

$$
\frac{1}{h_{d}} \int_{0}^{1} \int_{-\infty}^{t} K_{d}\left(\frac{s(x)-u}{h_{d}}\right) \mathrm{d} u \mathrm{~d} x=\int_{0}^{1} I\{s(x) \leq t\} \mathrm{d} x+o(1)
$$

and that for a positive kernel $K_{d}$ the function $s^{-1}\left(t, h_{d}\right)$ is always increasing with respect to $t$, independently whether the original function $s$ has this property, because

$$
\frac{\partial}{\partial t} s^{-1}\left(t, h_{d}\right)=\frac{1}{h_{d}} \int_{0}^{1} K_{d}\left(\frac{s(x)-t}{h_{d}}\right) \mathrm{d} x \geq 0 .
$$

For more details discussing the role of the inverse of $s^{-1}\left(t, h_{d}\right)$ as a monotone approximation of the function $s$ we refer to Sect. 2 in Dette et al. (2006).

In the present context we will use this concept to obtain monotone estimates of the variance function. For the sake of transparency we restrict ourselves to the problem of estimating an increasing variance function, the corresponding case of a decreasing variance is briefly mentioned in Remark 1. Observing the discussion in the previous paragraph we only need an unconstrained estimate of the variance function, and for this purpose we will use

$$
\hat{s}(x)=\frac{\sum_{i} K_{r}\left(\frac{x-x_{i}}{h_{r}}\right) \Delta_{i}^{2}}{\sum_{i} K_{r}\left(\frac{x-x_{i}}{h_{r}}\right)},
$$

where $K_{r}$ and $h_{r}$ denote a further kernel and bandwidth, respectively. We assume that the kernel $K_{r}$ is symmetric and has also compact support contained in the interval $[-1,1]$. In (6) the quantities $\Delta_{i}$ will denote residuals from a nonparametric fit (see e.g. Hall and Marron 1990) or pseudo residuals (see e.g. Rice 1984 or Gasser et al. 1986). For the sake of brevity we concentrate on the Nadaraya-Watson estimate based on smoothing squared residuals, but other types of estimators could be considered as well (see Remark 3 for some examples). Estimators of the form (6) have been considered by several authors, including Müller and Stadtmüller (1987, 1993), who mainly discussed pseudo residuals, Hall and Carroll (1989) and Akritas and Van Keilegom (2001), who proposed to use residuals from a nonparametric fit. Different smoothing 
techniques in the context of estimating the conditional variance have been proposed by Ruppert et al. (1997), Fan and Yao (1998) and Yu and Jones (2004).

Following our general motivation for constructing an increasing variance function estimate we propose the statistic

$$
\hat{s}_{I}^{-1}(t)=\frac{1}{N h_{d}} \sum_{i=1}^{N} \int_{-\infty}^{t} K_{d}\left(\frac{\hat{s}\left(\frac{i}{N}\right)-u}{h_{d}}\right) \mathrm{d} u
$$

as an estimate of $s^{-1}$. The required monotone increasing estimate of the conditional variance is now obtained by a simple inversion of this function and will be denoted by $\hat{s}_{I}$ throughout this paper. Note that the estimator $\hat{s}_{I}^{-1}$ is equal to 1 and 0 if $t>\max _{i=1}^{N} \hat{s}\left(\frac{i}{N}\right)+h_{d}$ and $t<\min _{i=1}^{N} \hat{s}\left(\frac{i}{N}\right)-h_{d}$, respectively, and only for

$$
t \in\left[\underset{i=1}{N} \hat{s}\left(\frac{i}{N}\right), \max _{i=1}^{N} \hat{s}\left(\frac{i}{N}\right)\right]
$$

the inverse of the function $\hat{S}_{I}^{-1}$ is calculated. The properties of this estimate depend on the particular method used for the unconstrained variance function estimate $\hat{s}$, but we prove below that in all important cases the monotone increasing estimate $\hat{s}_{I}$ is asymptotically normal distributed and first order equivalent to the corresponding unconstrained estimate. Note that the integral in (4) has been replaced by a simple quadrature formula with equidistant nodes. Moreover, the estimate $\hat{s}_{I}^{-1}$ can be considered as an integrated density estimate based on the "data"

$$
\left\{\hat{s}\left(\frac{i}{N}\right) \mid i=1, \ldots, N\right\}
$$

and the number $N$ used in this density estimator does not necessarily coincide with the sample size $n$ used for the calculation of the unconstrained estimate. The indices " $r$ " and " $d$ " of the kernel functions $K_{r}$ and $K_{d}$ correspond to the phrase "regression" and "density", because we combine a regression with a density estimate to define the estimator in (7). In the following we will discuss the properties of the new estimate for two different types of residuals $\Delta_{i}$ separately.

Remark 1 If the variance function $s$ is supposed to be strictly decreasing the estimate can easily be modified as

$$
\hat{s}_{A}^{-1}(t):=\frac{1}{N h_{d}} \sum_{i=1}^{N} \int_{t}^{\infty} K_{d}\left(\frac{\hat{s}\left(\frac{i}{N}\right)-u}{h_{d}}\right) \mathrm{d} u
$$

and the antitone estimate of the conditional variance is obtained by the inversion of this function. 


\section{Monotone variance function in action}

3.1 Monotone variance function estimation with pseudo residuals

Following Hall et al. (1990) we define pseudo residuals by

$$
\Delta_{i}=\Delta_{i, n}=\sum_{j=0}^{r} d_{j} Y_{i+j},
$$

where the quantities $d_{0}, \ldots, d_{r}$ are given weights satisfying

$$
\sum_{j=0}^{r} d_{j}=0, \quad \sum_{j=0}^{r} d_{j}^{2}=1 .
$$

In this case the preliminary estimator of the variance function is defined by

$$
\hat{s}(x)=\frac{\sum_{i=1}^{n-r} K_{r}\left(\frac{x-x_{i}}{h_{r}}\right) \Delta_{i}^{2}}{\sum_{i=1}^{n-r} K_{r}\left(\frac{x-x_{i}}{h_{r}}\right)} .
$$

Two special choices of pseudo residuals are very popular and have been considered by Rice (1984) $\left[r=1, d_{0}=-d_{1}=1 / \sqrt{2}\right]$ and Gasser et al. (1986) $\left[r=2, d_{0}=d_{2}=1 / \sqrt{6}, d_{1}=-2 / \sqrt{6}\right]$, while some general properties of variance estimates based on pseudo residuals are discussed in Dette et al. (1998) in the case of a homoscedastic regression model. Throughout this paragraph we assume that the regression function is Lipschitz continuous of order $\gamma>\frac{1}{4}$, which allows us to replace the quantities $\Delta_{i}$ in (9) by their unobservable counterparts

$$
\Delta_{i}^{\varepsilon}=\Delta_{i, n}^{\varepsilon}=\sum_{j=0}^{r} d_{j} \sqrt{s\left(x_{i+j}\right)} \varepsilon_{i+j}
$$

with sufficiently accuracy (see the proofs in Sect. 5.1 of the Appendix). The main properties of isotone variance function estimators using pseudo residuals are summarized in the following theorem, for which we require some assumptions regarding the bandwidths $h_{d}, h_{r}$ and the number $N$ used in the definition of the statistic $\hat{s}_{I}^{-1}$, that is

$$
\begin{aligned}
& h_{r} \rightarrow 0, \quad h_{d} \rightarrow 0, \\
& n h_{d} \rightarrow \infty, \quad n h_{r} \rightarrow \infty \\
& \lim _{h_{d} \rightarrow 0, h_{r} \rightarrow 0} h_{r} / h_{d}=\infty \\
& n h_{r}^{5}=O(1), \quad n=O(N), \\
& \frac{1}{n h_{r} h_{d}^{2}}=o(1) .
\end{aligned}
$$


Theorem 1 Assume that the design density $f$ and the variance function $s$ are positive and twice continuously differentiable, that the regression function $m$ in the nonparametric regression model (1) is Lipschitz continuous of order $\gamma>1 / 4$ and that the assumptions (13)-(17) are satisfied. Let $\hat{s}_{I}$ denote the isotone estimate of the variance function s obtained as the inverse of the statistic (7) with the statistic (11) as preliminary estimate, then it follows that for every $t \in(0,1)$ with $s^{\prime}(t)>0$

$$
\sqrt{n h_{r}}\left(\hat{s}_{I}(t)-s(t)-\Gamma\left(h_{d}, h_{r}, t\right)\right) \stackrel{\mathcal{D}}{\Rightarrow} \mathcal{N}\left(0, \beta^{2}(t)\right),
$$

where the asymptotic bias and variance are given by

$$
\begin{aligned}
\Gamma\left(h_{d}, h_{r}, t\right) & =\kappa_{2}\left(K_{d}\right) \frac{s^{\prime \prime}(t)}{\left(s^{\prime}(t)\right)^{2}} h_{d}^{2}+\kappa_{2}\left(K_{r}\right)\left(\frac{s^{\prime \prime} f+2 s^{\prime} f^{\prime}}{f}\right)(t) h_{r}^{2}, \\
\beta^{2}(t) & =\frac{s^{2}(t)\left\{m_{4}(t)-1+4 \delta_{r}\right\}}{f(t)} \int_{-1}^{1} K_{r}^{2}(u) d u,
\end{aligned}
$$

respectively, for a given kernel $K$ the constant $\kappa_{2}(K)$ is defined as

$$
\kappa_{2}(K)=\frac{1}{2} \int_{-1}^{1} v^{2} K(v) \mathrm{d} v
$$

and the quantitiy $\delta_{r}$ is given by

$$
\delta_{r}=\sum_{k=1}^{r}\left(\sum_{j=0}^{r-k} d_{j} d_{j+k}\right)^{2} \quad(r \geq 1) .
$$

Remark 2 Note that the dominating term in the representation (19) for the bias is given by

$$
\Gamma\left(h_{d}, h_{r}, t\right)=\kappa_{2}\left(K_{r}\right)\left(\frac{s^{\prime \prime} f+2 s^{\prime} f^{\prime}}{f}\right)(t) h_{r}^{2}+o\left(h_{r}^{2}\right),
$$

because $h_{d}=o\left(h_{r}\right)$ by assumption (15). In the context of estimating a monotone regression function it was observed by Dette et al. (2006) by a numerical study that the choice of the bandwidth $h_{d}$ in the density step is less critical compared to the choice of the bandwidth $h_{r}$ in the regression step. The same fact is true for the problem of estimating the conditional variance. A simple calculation shows that the choice $h_{d}=h_{r}^{\alpha}$ with $\alpha \in(1,2)$ satisfies the assumptions (13)-(17) in Theorem 1 and the approximation (23) is well justified. However, an extensive numerical study shows that there exist no substantial differences with respect to the performance of the estimate $\hat{s}_{I}$ for larger values of $\alpha$. Based on our numerical experience we recommend to choose $h_{d}$ as $h_{d}=h_{r}^{\alpha}$ for some $\alpha \geq 1.5$.

Remark 3 It follows from the proof of Theorem 1 that the choice of a different smoothing procedure in (6) does not change the asymptotic variance of the 
resulting monotone estimate of the variance function, but its asymptotic bias. For example, if a local linear estimate (see Fan and Gijbels 1996) is applied to the squared pseudo residuals (9), then the resulting estimate $\hat{s}_{I}$ is asymptotically normal distributed, that is

$$
\sqrt{n h_{r}}\left(\hat{s}_{I}(t)-s(t)-\Gamma_{\mathrm{loc}}\left(h_{d}, h_{r}, t\right)\right) \stackrel{\mathcal{D}}{\Rightarrow} \mathcal{N}\left(0, \beta^{2}(t)\right)
$$

where the asymptotic variance is given by (20) and the bias is defined by

$$
\Gamma_{\mathrm{loc}}\left(h_{d}, h_{r}, t\right)=\kappa_{2}\left(K_{d}\right) \frac{s^{\prime \prime}(t)}{\left(s^{\prime}(t)\right)^{2}} h_{d}^{2}+\kappa_{2}\left(K_{r}\right) s^{\prime \prime}(t) h_{r}^{2}=\kappa_{2}\left(K_{r}\right) s^{\prime \prime}(t) h_{r}^{2}+o\left(h_{r}^{2}\right) .
$$

Other estimates for the regression step can be treated similarly. For example, if the local log-linear estimator proposed by $\mathrm{Yu}$ and Jones (2004) is used as preliminary unconstrained estimate of the conditional variance, the isotonized estimate $\hat{s}_{I}$ has still asymptotic variance $\beta^{2}(t) / n h_{r}$, asymptotic bias is given by

$$
\Gamma_{Y J}\left(h_{d}, h_{r}, t\right)=\kappa_{2}\left(K_{d}\right) \frac{s^{\prime \prime}(t)}{\left(s^{\prime}(t)\right)^{2}} h_{d}^{2}+\kappa_{2}\left(K_{r}\right)\left(s^{\prime \prime}(x)-\frac{\left(s^{\prime}(x)\right)^{2}}{s(x)}\right) h_{r}^{2}+o\left(h_{r}^{2}\right)
$$

and the appropriately standardized version of $\hat{s}_{I}$ is asymptotically normal distributed.

Remark 4 For the different estimates of the variance function considered in Theorem 1 and Remark 3 it follows from the results of Müller and Stadtmüller (1993), Yu and Jones (2004) and the proof of Theorem 1 that the isotone estimates of the variance function are first order asymptotically equivalent to the unconstrained estimates in the sense that they yield the same first order expansion for the bias and variance.

Remark 5 Note that the asymptotic variance in Theorem 1 depends on the constant $\delta_{r}$ defined in (22). For the estimator of Rice (1984) we have $r=1$, $d_{0}=-d_{1}=1 / \sqrt{2}$, which yields $\delta_{1}=d_{0}^{4}=1 / 4$ and

$$
\beta_{R}^{2}(t)=\frac{s^{2}(t) m_{4}(t)}{f(t)} \int_{-1}^{1} K_{r}^{2}(u) \mathrm{d} u .
$$

A different weighting scheme was suggested by Gasser et al. (1986), who used for a uniform design $\left(d_{0}, d_{1}, d_{2}\right)=\frac{1}{\sqrt{6}}(1,-2,1)$ in the context of a nonparametric homoscedastic regression model, and argued that this sequence yields a smaller bias in the approximation of the pseudo residuals by the quantities defined in (12). For this choice we obtain in Theorem $1(r=2) \delta_{2}=17 / 36$,

$$
\beta_{G}^{2}(t)=\frac{s^{2}(t)}{f(t)}\left(m_{4}(t)+\frac{8}{9}\right) \int_{-1}^{1} K_{r}^{2}(u) \mathrm{d} u .
$$


Alternatively one could try to minimize the asymptotic variance (20) by an appropriate choice of the weights $d_{0}, \ldots, d_{r}$. Hall et al. (1990) determined for a fixed order $r$ optimal weights $d_{j}$ such that the quantity $\delta_{r}$ in (22) becomes minimal (see Table 1 of their paper). For this choice we have

$$
\sum_{j=0}^{r-k} d_{j} d_{j+k}=-\frac{1}{2 r}
$$

the minimal value of $\delta_{r}$ is obtained as $\delta_{r}{ }^{\text {opt }}=1 / 4 r$ and the resulting asymptotic variance is given by

$$
\beta_{\mathrm{opt}}^{2}(t)=\frac{s^{2}(t)}{f(t)}\left(m_{4}(t)-\frac{r-1}{r}\right) \int_{-1}^{1} K_{r}^{2}(u) \mathrm{d} u .
$$

Consequently the asymptotic variance in Theorem 1 can be decreased by using an optimal difference sequence in the sense of Hall et al. (1990) and an increasing order $r$. However, some care is appropriate in these asymptotic considerations. For realistic sample sizes it is also necessary to obtain a sufficiently small bias of the pseudo residuals $\Delta_{i}$ and optimal sequences usually produce a small variance but a large bias. The general choice of the weights in the definition of the pseudo residuals was carefully discussed by Dette et al. (1998) in the context of homoscedastic nonparametric regression. These authors give some data driven guidelines for choosing an appropriate order $r$ and the corresponding weights $d_{0}, \ldots, d_{r}$. In general difference sequences for $r=1$ or $r=2$ will be sufficient and the improvement in efficiency by using a larger order is negligible in most cases (compare also with the results of our simulation study in Sect. 4).

\subsection{Monotone variance function estimation with nonparametric residuals}

Following Hall and Marron (1990) we consider residuals

$$
\hat{\varepsilon}_{i}=Y_{i}-\hat{m}\left(x_{i}\right)
$$

where

$$
\hat{m}(x)=\frac{\sum_{i=1}^{n} K\left(\frac{x-x_{i}}{h}\right) Y_{i}}{\sum_{i=1}^{n} K\left(\frac{x-x_{i}}{h}\right)}
$$

is the Nadaraya-Watson estimate of the regression function. The unconstrained estimate of the conditional variance is now given by

$$
\hat{s}(x)=\frac{\sum_{i=1}^{n} K_{r}\left(\frac{x-x_{i}}{h_{r}}\right) \hat{\varepsilon}_{i}^{2}}{\sum_{i=1}^{n} K_{r}\left(\frac{x-x_{i}}{h_{r}}\right)} .
$$


Note that different bandwidths are used for the estimation of the regression and variance function and that the kernels used in (29) and (30) do not necessarily coincide. The following result is an analogue of Theorem 1 for the case, where residuals from a nonparametric fit are used in the construction of a monotone estimate of the conditional variance. For its proof we require the following assumption regarding the bandwidth $h$ in the Nadraya-Watson estimate (29)

$$
h \rightarrow 0, \quad n h \rightarrow \infty, \quad h_{r}=O(h) .
$$

Theorem 2 Assume that the design density $f$ and the variance function $s$ are positive, that $f, s$ and the regression function $m$ in the nonparametric regression model (1) are twice continuously differentiable and that the assumptions (13)-(17) and (31) are satisfied. Let $\hat{s}_{I}$ denote the isotone estimate of the variance function s obtained as the inverse of the statistic (7) with the statistic (30) as preliminary estimate, then it follows that for every $t \in(0,1)$ with $s^{\prime}(t)>0$

$$
\sqrt{n h_{r}}\left(\hat{s}_{I}(t)-s(t)-\Gamma\left(h_{d}, h_{r}, t\right)\right) \stackrel{\mathcal{D}}{\Rightarrow} \mathcal{N}\left(0, \delta^{2}(t)\right),
$$

where the asymptotic bias is defined by (19) and the asymptotic variance is given by

$$
\delta^{2}(t)=\frac{s^{2}(t)\left\{m_{4}(t)-1\right\}}{f(t)} \int_{-1}^{1} K_{r}^{2}(u) \mathrm{d} u .
$$

Note that the asymptotic bias of the monotone estimates based on (11) and (30) coincide, while there is a difference in the asymptotic variance. The asymptotic variance in (33) can be considered as a limit $(r \rightarrow \infty)$ of the asymptotic variance of the monotone estimate using pseudo residuals with an optimal difference sequence. We note, however, that for realistic sample sizes these asymptotic differences are rarely observable.

Remark 6 A different choice of the estimator $\hat{m}$ (for example a local polynomial or the Gasser-Müller estimator) does not change the asymptotic result in Theorem 2. On the other hand, if a different estimator is used for the smoothing of the squared residuals in (30) the asymptotic bias has to be modified appropriately (compare with Remark 3). Moreover, it can be shown by similar arguments as given in Fan and Yao (1998) that the estimates $\hat{s}_{I}$ considered in Theorem 2 and its corresponding preliminary estimate $\hat{s}$ defined in (30) are first order asymptotically equivalent.

\subsection{Extension to other models}

The results discussed so far remain valid (subject to an appropriate modification of the constants) for other nonparametric regression models. As an illustration consider the stochastic regression model 


$$
Y_{i}=m\left(X_{i}\right)+\sqrt{s\left(X_{i}\right)} \varepsilon_{i},
$$

where $\left(X_{i}, Y_{i}\right)_{i \in \mathbb{Z}}$ is a strictly stationary two dimensional process with $E\left[Y_{i} \mid\right.$ $\left.X_{i}=x\right]=m(x), \operatorname{Var}\left(Y_{i} \mid X_{i}=x\right)=s(x) \neq 0, E\left[\varepsilon_{i}^{4} \mid X_{i}=x\right]=m_{4}(x)$. Fan and Yao (1998) proposed $\tilde{s}(x)=\hat{\alpha}$ as estimate of the conditional variance, where

$$
(\hat{\alpha}, \hat{\beta})=\underset{\alpha, \beta}{\operatorname{argmin}} \sum_{i=1}^{n}\left\{\hat{r}_{i}-\alpha-\beta\left(X_{i}-x\right)\right\}^{2} K_{r}\left(\frac{X_{i}-x}{h_{r}}\right)
$$

is the local linear estimate based on the nonparametric residuals $\hat{r}_{1}, \ldots, \hat{r}_{n}$. These quantities are defined by $\hat{r}_{j}=Y_{j}-\hat{a}$, where

$$
(\hat{a}, \hat{b})=\underset{a, b}{\operatorname{argmin}} \sum_{i=1}^{n}\left\{Y_{i}-a-b\left(X_{i}-X_{j}\right)\right\}^{2} K\left(\frac{X_{i}-X_{j}}{h}\right)
$$

is the local linear estimate of the regression function (and its derivative) at the point $X_{j}$. If $\tilde{s}_{I}$ denotes the isotonization of the conditional variance estimate obtained as the inverse of the statistic (7) with $\hat{s}=\tilde{s}$, the assumptions of Theorem 2 and the conditions 1-5 in Appendix 1 of Fan and Yao (1998) are satisfied, then the statistic

$$
\sqrt{n h_{r}}\left\{\tilde{s}_{I}(x)-s(x)-\Gamma_{\mathrm{loc}}\left(h_{d}, h_{r}, x\right)\right\}
$$

is asymptotically normal with mean 0 and variance $\delta^{2}(x)$ defined in (33), where the quantity $\Gamma_{\text {loc }}\left(h_{d}, h_{r}, x\right)$ is given by (25) and $f$ is the marginal density of $X$. Again the monotonized estimate is first order asymptotically equivalent to the unconstrained estimate (see Fan and Yao 1998, Theorem 1).

\subsection{Confidence intervals}

As pointed out by a referee the results of the paper can easily be used to obtain confidence intervals for a monotone conditional variance. For the sake of completeness we briefly indicate the construction in the situation considered in Theorem 2 where the fourth moment does not depend on $t$, i.e. $m_{4}(t)=m_{4}$ and $h_{r}=o\left(n^{-1 / 5}\right)$. In this case the statement in (32) reduces to

$$
\sqrt{n h_{r}}\left(\hat{s}_{I}(t)-s(t)\right) \stackrel{\mathcal{D}}{\Rightarrow} \mathcal{N}\left(0, \delta^{2}(t)\right),
$$

and an estimate of the asmyptotic variance $\delta^{2}(t)$ is obtained as

$$
\hat{\delta}^{2}(t)=\frac{\hat{s}_{I}^{2}(t)\left(\hat{m}_{4}-1\right)}{\hat{f}(t)} \int_{-1}^{1} K_{r}^{2}(u) \mathrm{d} u,
$$


where $\hat{f}$ is the (non random) density estimate from the predictors $\left\{x_{i, n}\right\}_{i=1, \ldots, n}$,

$$
\begin{aligned}
\hat{m}_{4} & =\frac{\hat{A}_{4, n}}{\hat{S}_{4, n}^{2}}-3 \\
\hat{A}_{4, n} & =\frac{1}{2(n-1)} \sum_{j=2}^{n}\left(\Delta_{j}^{*}\right)^{4} \\
\hat{S}_{4, n} & =\frac{1}{4(n-3)} \sum_{j=3}^{n}\left(\Delta_{j}^{*}\right)^{2}\left(\Delta_{j-2}^{*}\right)^{2}
\end{aligned}
$$

and $\Delta_{j}^{*}=Y_{j}-Y_{j-1}, j=2, \ldots, n$ (see e.g. Dette and Munk 1998). Therefore an asymptotic $(1-\alpha)$-confidence interval for $s(t)$ is given by

$$
\left[\hat{s}_{I}(t)-z_{1-\alpha / 2} \frac{\hat{\delta}(t)}{\sqrt{n h_{r}}}, \hat{s}_{I}(t)+z_{1-\alpha / 2} \frac{\hat{\delta}(t)}{\sqrt{n h_{r}}}\right]
$$

where $z_{1-\alpha / 2}$ denotes the $(1-\alpha / 2)$-quantile of the standard normal distribution. Note that the pseudo residuals $\Delta_{j}^{*}$ correspond to the choice $r=1$ in (9) and that other choices would result in different estimates of $m_{4}$. The general case of a non constant function $m_{4}$ can be obtained by calculating $\hat{m}_{4}$ locally. Bandwidths of optimal order, i.e. $h_{r}=c n^{-1 / 5}$, could also be used, if the bias in Theorem 2 is estimated appropriately. The details are omitted for the sake of brevity.

\section{Finite sample properties}

In this section we illustrate the finite sample properties of the monotone estimates of the conditional variance by means of a small simulation study and a data example is analyzed using the new methodology.

\subsection{A small simulation study}

We begin with a comparison of different estimates based on pseudo residuals (see Sect. 3.1) and then compare the best estimates in this class with the monotone variance estimates based on nonparametric residuals (see Sect. 3.2). For the sake of brevity we restrict our study to two regression models, that is

$$
\begin{aligned}
& Y_{i}=\sin \left(6 x_{i}\right)+\sqrt{\frac{3}{2} x_{i}^{2}} \varepsilon_{i} ; \quad i=1, \ldots, n \\
& Y_{i}=x_{i}+\sqrt{x_{i}} \varepsilon_{i} ; \quad i=1, \ldots, n
\end{aligned}
$$


where $\varepsilon_{1}, \ldots, \varepsilon_{n}$ i.i.d. $\sim \mathcal{N}(0,1)$ and the sample size is $n=100$. As a design a uniform design $(f(x)=1)$ is considered, while the Epanechnikov kernel is used for the kernels $K_{d}$ and $K_{r}$ in the density and regression estimate. Note that for this kernel the integral in (7) can be evaluated explicitly, and that there are several other kernels (e.g. beta-kernels), where no numerical integration is required in (7).

The bandwidth $h_{d}$ for the density step is always given by $h_{d}=\hat{h}_{r}^{1.9}$, where $\hat{h}_{r}$ is a data driven bandwidth of order $n^{-1 / 5}$, which will be specified below. We applied 2000 simulation runs to calculate the squared bias, variance and mean squared error of the different estimates in the interval $[0,1]$.

\subsubsection{Finite sample properties of difference based estimates}

In order to avoid boundary effects we use a local linear estimate based on the pseudo residuals (9) in the regression step (for a definition of this estimate see also Sect. 3.3), where different orders $r$ and different sequences of weights are investigated. The choice of the bandwidth is important for the performance of the estimate and we use the following simple plug-in-rule

$$
\hat{h}_{r}=\left(\frac{\hat{A}}{n}\right)^{1 / 5}
$$

where

$$
\hat{A}=\frac{1}{n-r} \sum_{i=1}^{n-r}\left(\Delta_{i}^{2}-\bar{\Delta}^{2}\right)^{2}
$$

is the empirical variance of the pseudo residuals $\Delta_{1}^{2}, \ldots, \Delta_{n}^{2}\left(\bar{\Delta}^{2}=\frac{1}{n-r} \sum_{i=1}^{n-r} \Delta_{i}^{2}\right)$. Because $\hat{A}$ is a consistent estimate of

$$
\frac{1}{n} \sum_{i=1}^{n} \operatorname{Var}\left(\Delta_{i}^{2}\right) \approx \int_{0}^{1} s^{2}(x)\left\{2+\left(m_{4}(x)-3\right) \sum_{\ell=0}^{r} d_{\ell}^{4}\right\} f(x) \mathrm{d} x
$$

the bandwidth (37) is (asymptotically) proportional to the global (with respect to the integrated mean squared error criterion) optimal bandwidth, if a local linear estimate is applied to the pseudo residuals $\Delta_{1}^{2}, \ldots, \Delta_{n}^{2}$. Smoothing parameters proportional to locally optimal bandwidths could be obtained similarly, but the bandwidth (37) yields reasonable results in all cases considered in our study.

In what follows we display the mean squared error, squared bias and variance curves of various estimates for the variance function. It should be noted that these curves depend on the specific choice of $h_{d}$, because they are depicted on a very fine scale. The effect of $h_{d}$ on the curve estimate is less visible. Our first example investigates the optimal difference sequences introduced by Hall et al. (1990), which minimize the asymptotic variance of the monotone estimate $\hat{s}_{I}$. In Fig. 1 we show the curves of the mean squared error, squared bias and variance 

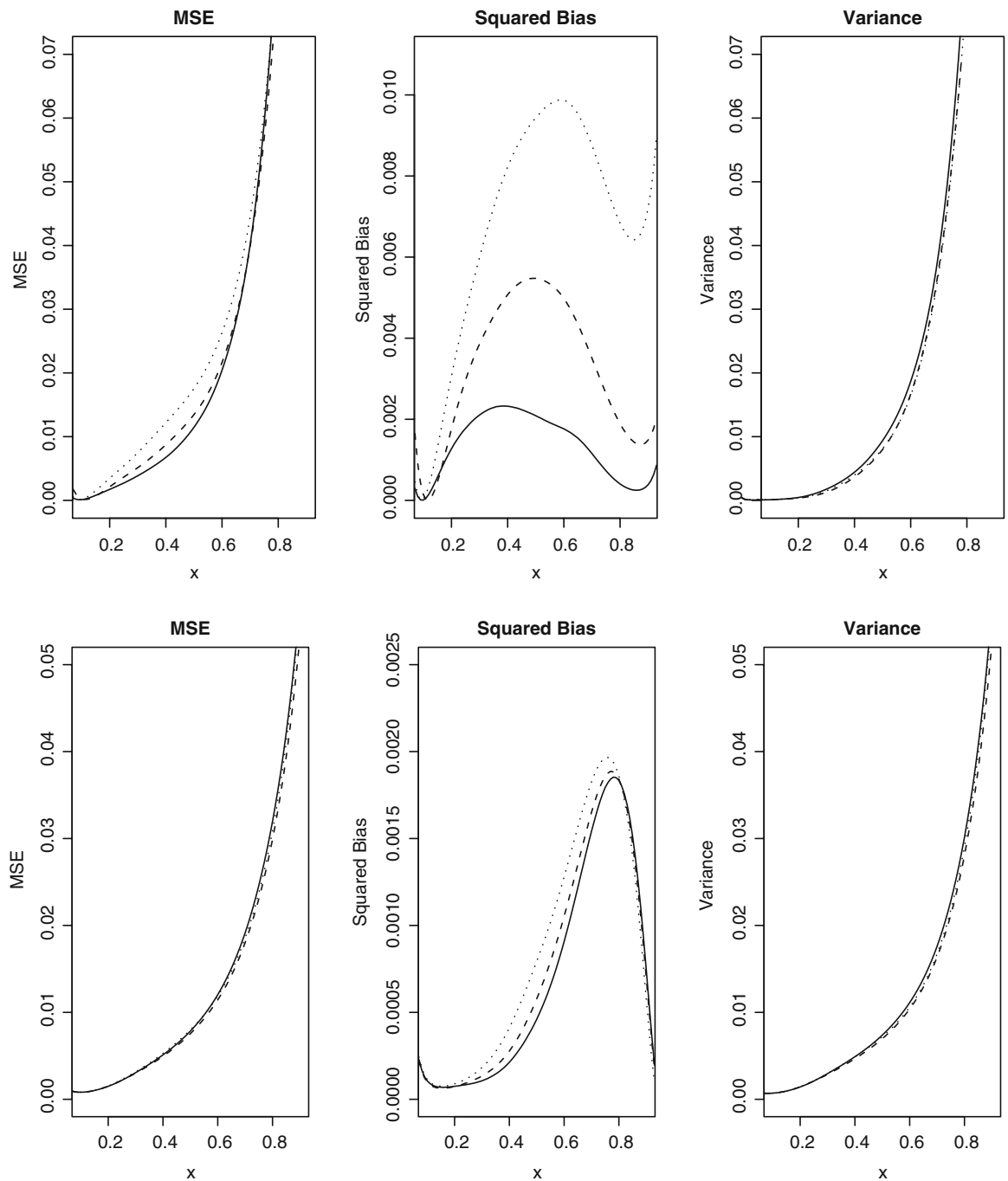

Fig. 1 Simulated mean squared error, squared bias and variance of the monotone variance estimate (7) based on pseudo residuals with an optimal difference sequence proposed by Hall et al. (1990); $r=1$ : solid line; $r=2:$ dashed line; $r=3:$ dotted line. The upper panel corresponds to model (35) and the lower panel to model (36)

with an optimal difference sequence of order $r=1,2,3$. Variance estimates based on pseudo residuals with an optimal difference of larger order show a very similar picture and are therefore not depicted.

We observe that for model (35) all estimates behave very similary with respect to the variance criterion (with slight advantage for difference sequences of order $r=2,3$ ) and that the variance of the estimate $\hat{s}_{I}$ is strictly increasing. This reflects the asymptotic representation in Theorem 1, which shows that the variance must be proportional to 


$$
\left(n h_{r}\right)^{-1} \cdot\left(2+\frac{1}{r}\right) \cdot \frac{3}{2} \cdot t^{2} \cdot 0.6
$$

(recall that $f(x) \equiv 1$ and that for the Epanechnikov kernel $\int K^{2}(u) \mathrm{d} u=3 / 5$ ). On the other hand there are advantages with respect to the squared bias criterion for the estimates using pseudo residuals with a lower order $(r=1,2)$, while the monotone variance estimate based on pseudo residuals with an optimal difference sequence of order 3 has a substantial larger bias. A similar phenomenon was observed by Dette and Munk (1998) in the context of variance estimation in a homoscedastic nonparametric regression model. These differences are also reflected in the mean squared error curves, where the estimates with pseudo residuals of order one and two have the best performance.

Note that for the regression model (36) the second derivative of the variance function vanishes, which results in a substantially smaller bias in Theorem 1 . As a consequence the variance has a stronger impact on the mean squared error and we expect that variance estimates based on optimal difference sequences of larger order have a better performance. These asymptotic properties are partially reflected in the squared bias curve (see the lower panel of Fig. 1). On the other hand there are no substantial differences between the variance estimates $\hat{s}_{I}$ based on pseudo residuals with optimal difference sequences of different order and the variance dominates the mean squared error. The best performance with respect to the mean squared error criterion and the differences between the three estimates are now mainly caused by the variance.

Figure 2 shows the corresponding curves for model (35) and (36) if the difference sequence

$$
d_{i}=(-1)^{i} \frac{\left(\begin{array}{l}
r \\
i
\end{array}\right)}{\left(\begin{array}{c}
2 r \\
r
\end{array}\right)^{1 / 2}} \quad r=1,2,3
$$

is used for the construction of the pseudo residuals $\Delta_{i}$ in (9). As pointed out by Dette et al. (1998) these difference sequences reduce the bias at the cost of a larger variance. Note that for $r=1$ and $r=2$ this choice yields the difference sequences proposed by Rice (1984) and Gasser et al. (1986), respectively. For order $r=3$ this effect is clearly visible in model (35), where we observe a slightly smaller curve for the squared bias (compare also the upper panels in Figs. 1 and 2), but a larger variance. For both models the difference sequence with $r=1$ has the best performance in the class (39) and the decrease with respect to the bias does not compensate the increase in variance.

In model (35) the estimate with a difference sequence of order $r=1$ produces the smallest mean squared error curve among the estimates using difference sequences of the form (39) (see the upper panel Fig. 2), but the estimate with an optimal difference sequence of order $r=2$ has a similar mean squared error (see Fig. 1 and note that for $r=1$ the optimal difference sequence and the difference sequence of the form (39) coincide). In model (36) the best optimal difference sequence (obtained by using the order $r=2$ or $r=3$ ) yields a smaller mean squared error than the best difference sequence from the class (39). 

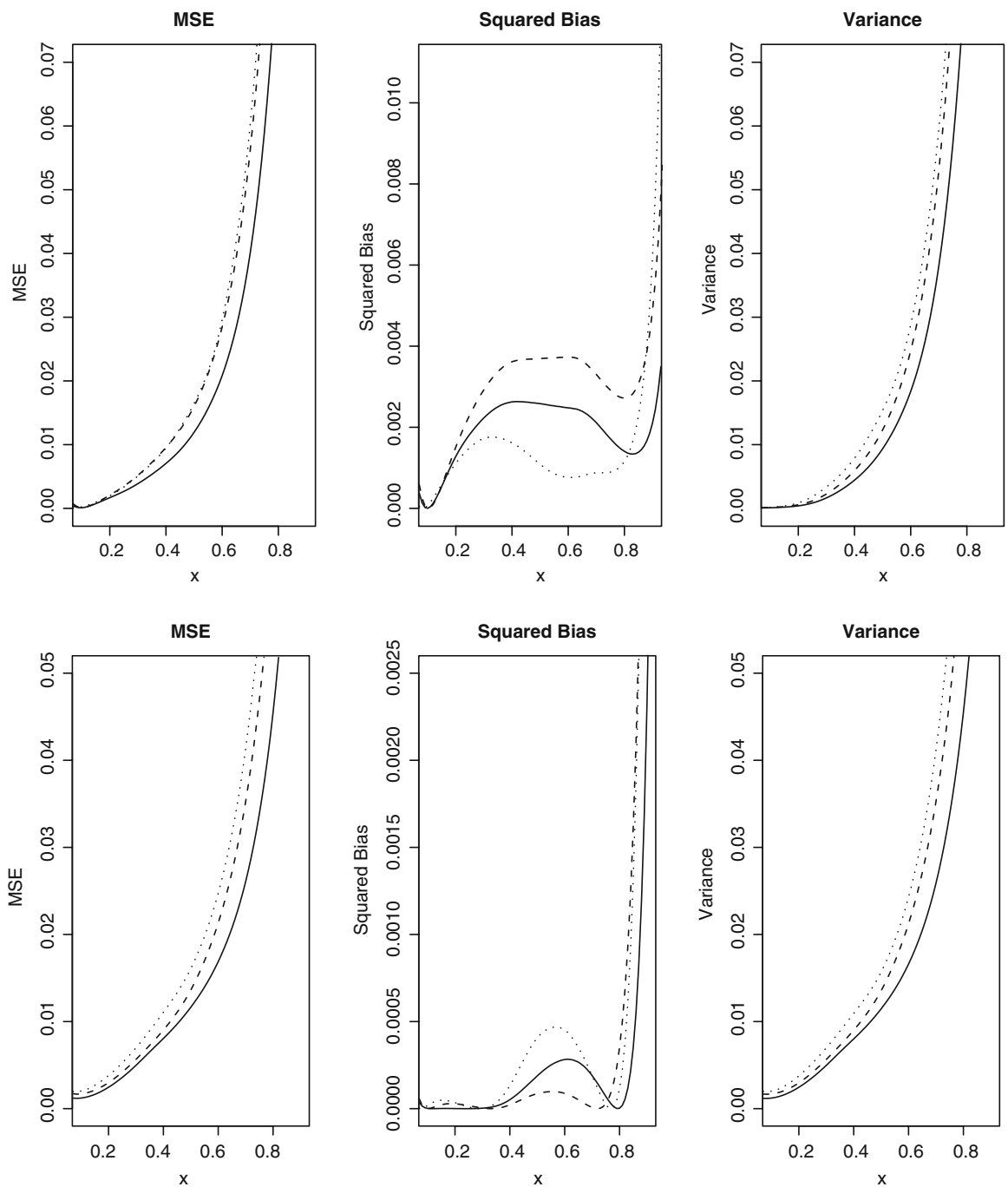

Fig. 2 Simulated mean squared error, squared bias and variance of the monotone variance estimate (7) based on pseudo residuals with a difference sequence of the form (39); $r=1$ : solid line; $r=2:$ dashed line; $r=3:$ dotted line. The upper panel corresponds to model (35) and the lower panel to model (36)

Variance estimates based on pseudo residuals with optimal difference sequences produce a substantially smaller variance and mean squared error compared to the estimators using the difference sequences of the form (39). Because other simulation results (which are not depicted here for the sake of brevity) show a similar picture we recommend the use of the optimal difference sequences if pseudo residuals are used in the construction of the monotone estimate $\hat{s}_{I}$ of the conditional variance. We now compare these estimates with 
the monotone variance estimates based on nonparametric residuals introduced in Sect. 2.2.

\subsubsection{Pseudo or nonparametric residuals?}

For the construction of the nonparametric residuals $\hat{\varepsilon}_{i}=Y_{i}-\hat{m}\left(x_{i}\right)$ we use a local linear estimate $\hat{m}$ with bandwidth

$$
h=\left(\frac{\hat{\sigma}^{2}}{n}\right)^{1 / 5}
$$

where $\hat{\sigma}^{2}=\frac{1}{2(n-1)} \sum_{i=2}^{n}\left(Y_{i}-Y_{i-1}\right)^{2}$ is the nonparametric estimate of Rice (1984) for the integrated variance. Again a local linear estimate based on the nonparametric residuals $\hat{\varepsilon}_{1}^{2}, \ldots, \hat{\varepsilon}_{n}^{2}$ is used in the preliminary regression step. The bandwidth $h_{r}$ was chosen according to the plug-in rule (37) where the pseudo residuals $\Delta_{i}^{2}$ in (38) are now replaced by the nonparametric residuals $\hat{\varepsilon}_{i}^{2}$.

Throughout this section monotone variance estimators obtained from the nonparametric residuals $\hat{\varepsilon}_{1}^{2}, \ldots, \hat{\varepsilon}_{n}^{2}$ will be denoted by $\hat{s}_{I}^{N}$, while the estimates obtained from pseudo residuals with the best optimal variance sequence $(r=2)$ and the best sequence of the form (39) $(r=1)$ are denoted by $\hat{s}_{I}^{D_{2}}$ and $\hat{s}_{I}^{D_{1}}$, respectively. Note that in the case $r=1$ the optimal difference sequence and the difference sequence of the form (39) coincide. For both models (35) and (36) we observe in Fig. 3 that the estimate $\hat{s}_{I}^{N}$ has the smallest variance followed by $\hat{s}_{I}^{D_{2}}$ and $\hat{s}_{I}^{D_{1}}$. This corresponds to asymptotic theory, which shows that the asymptotic variance of the statistics $\hat{s}_{I}^{N}, \hat{s}_{I}^{D_{1}}, \hat{s}_{I}^{D_{2}}$ is given by

$$
\frac{6}{5} \frac{s^{2}(t)}{n h_{r}}, \frac{9}{5} \frac{s^{2}(t)}{n h_{r}}, \frac{3}{2} \frac{s^{2}(t)}{n h_{r}}
$$

respectively. However, Fig. 3 also shows that there are differences in the behaviour with respect to the squared bias criterion. In both models the estimate $\hat{s}_{I}^{N}$ produces the largest bias [but this is negligible in the model (36)]. The estimate $\hat{s}_{I}^{D_{1}}$ has a smaller (squared) bias in both models than $\hat{s}_{I}^{D_{2}}$. In model (35) the estimates based on pseudo residuals have a smaller mean squared error than $\hat{s}_{I}^{N}$ over a broad range of the interval $[0,1]$. Only at the right boundary of the interval $[0,1]$ the smaller variances of $\hat{s}_{I}^{N}$ compensate its larger bias, such that it becomes the best estimate in our comparison. On the other hand in model (36) the bias can be neglected and the mean squared error is dominated by the variance. As a consequence the monotone variance estimate $\hat{s}_{I}^{N}$ based on nonparametric residuals yields the smallest mean squared error for the complete interval $[0,1]$. 

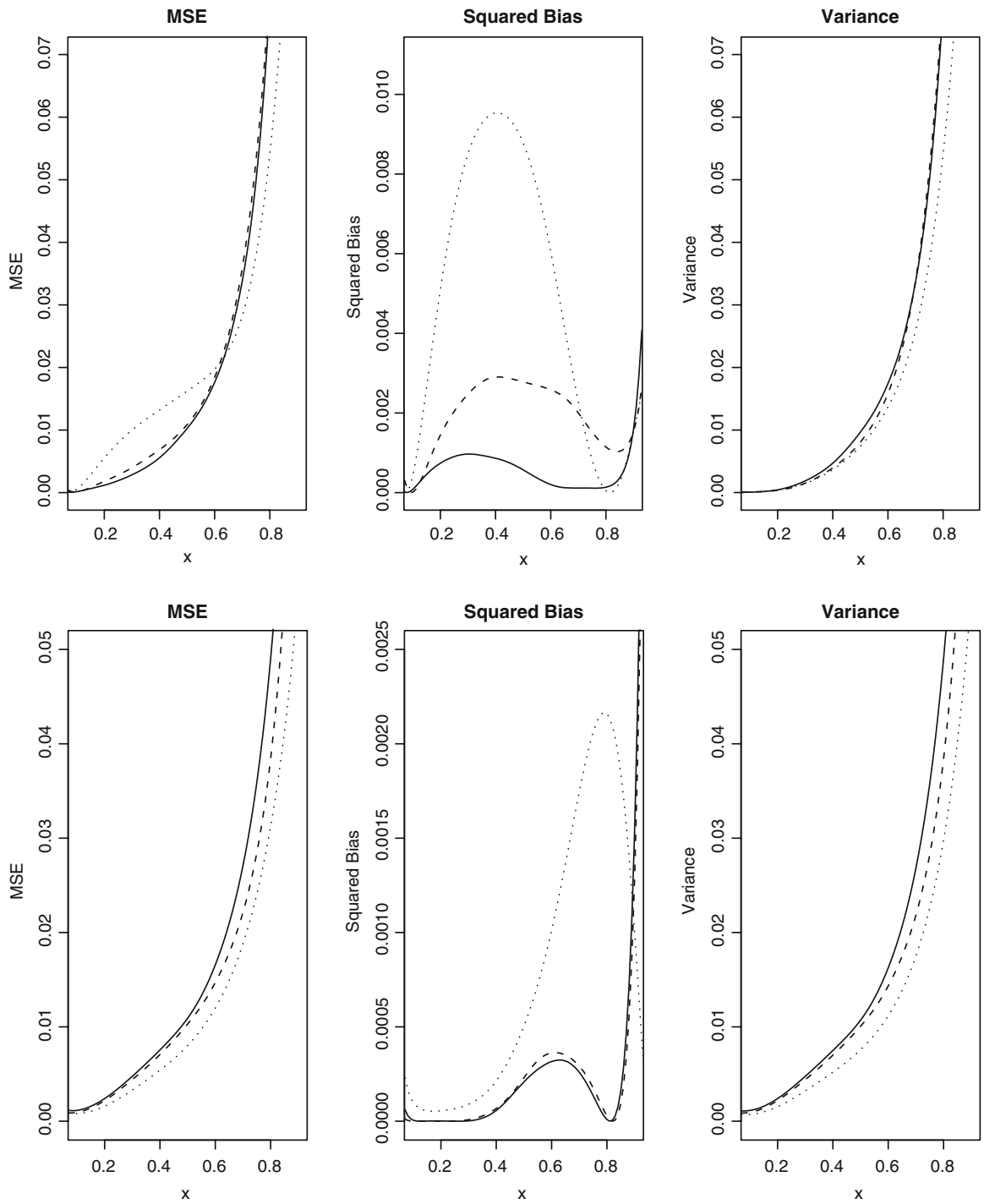

Fig. 3 Simulated mean squared error, squared bias and variance of the monotone variance estimate (7) based on pseudo residuals with an optimal difference sequence order $r=1$ (solid line), with an optimal difference sequence of order $r=2$ (dashed line) and based on nonparametric residuals (dotted line). The upper panel corresponds to model (35) and the lower panel to model (36)

\subsection{A data example}

We conclude this section with an application of the monotone variance estimator to real data. The data set consists of 4177 records of the mollusc Abalone (genus Haliotis) with variables concerning several measurements of lengths and weights as well as the categorical variable gender. Further, the number of 
rings in the shell has been counted, from which by adding 1.5 the age of the mollusc can be derived (for the original study see Nash et al. 1994). 15 (obvious) outliers have been omitted in the following data analysis.

In the left panel of Fig. 4 the scatterplot of the variables age (in years) and weight of the shell (in grams) is displayed, and it appears that both the mean function and the variance function are monotone.

The quadratic fit in the right panel of Fig. 4 (dotted line) shows large deviations of the curve at the boundary, such that a quadratic regression model seems to be inappropriate for these data. The local-linear estimator produces a more sophisticated figure. After a short flat beginning, the slope of the curve increases between the age of 5 and 12 years, passes to a moderate rise up to the age 25 and forms a peak thereafter, though this final increase is less confident

Fig. 4 Left panel: Scatterplot of age (in years) and shell weight (in grams) of the mollusc Abalone (Haliotis). Right panel: A parametric quadratic fit (dotted line), a nonparametric local-linear fit (dashed line) and a nonparametric monotone fit (solid line) of the mean function
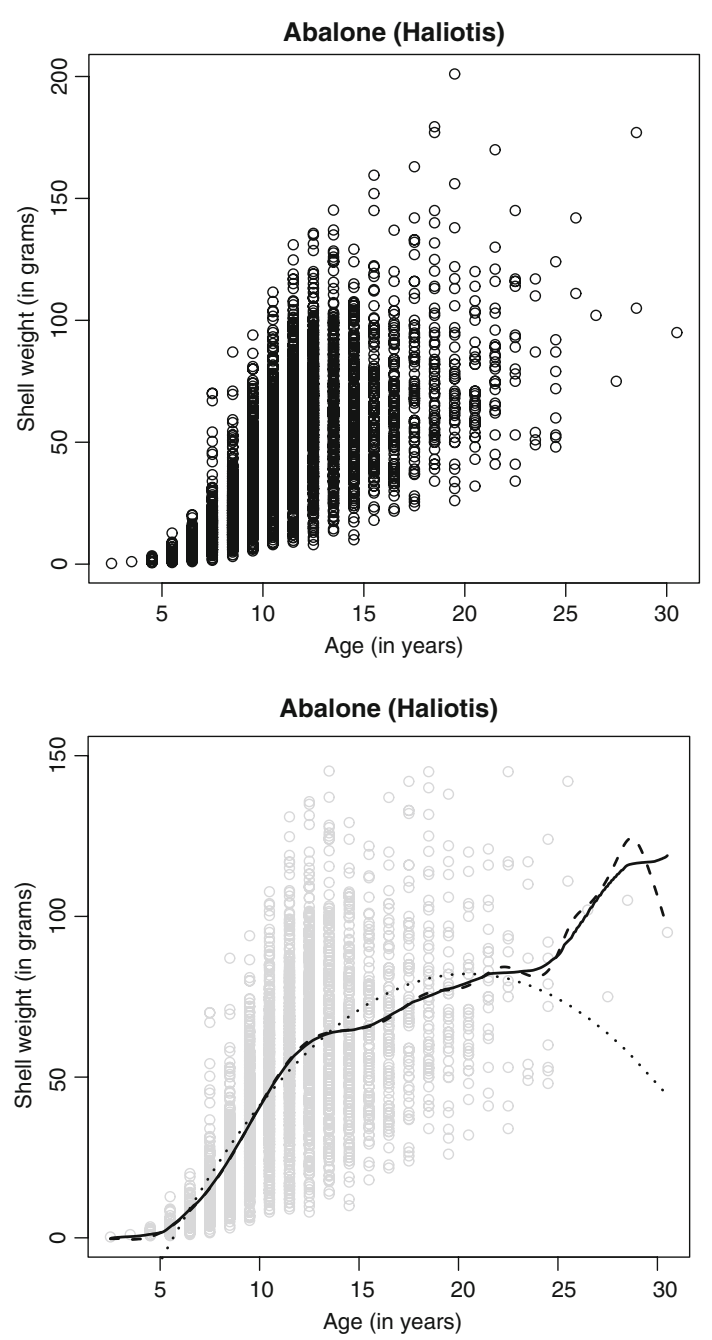
because of the sparse data. For the most ages the parametric and nonparametric fit exhibit substantial differences. The solid curve shows a monotonization of the local-linear fit, which has been carried out by the same methods as for the monotone variance $\hat{s}_{I}$ discussed in this article (see Dette et al. 2006). The monotone estimator eliminates the two decreasing parts of the unconditional estimator between 22.5 and 24.5 years and after 28.5 years.

Turning to the estimation of the variance function, the left panel in Fig. 5 shows the estimated squared residuals $\hat{\varepsilon}_{i}^{2}=\left(Y_{i}-\hat{m}_{I}\left(x_{i}\right)\right)^{2}$, where $\hat{m}_{I}$ is the isotonization of the local-linear estimator (see the right panel of Fig. 4). Here a monotone relation is less apparent than in the scatterplot of age versus shell weight. But this might be a visual effect, since the local-linear estimate in the right panel (dashed line) shows no substantial decrease up to the age 22, where the data become sparse. This visual effect seems to be generated by fewer extreme squared residuals after an age of 20 , but increasing variance among the smaller ones in these ages. The monotone estimation of the variance function in the right panel (solid line) shows a similar shape as the unconstrained estimator, but eliminates decreasing parts, especially between the age of 18 and 25 .

\section{Proofs}

\subsection{Proof of Theorem 1}

The proof is performed in several steps. At first we calculate the asymptotic bias and variance of the statistic $\hat{s}_{I}^{-1}$ defined in (7), secondly, we establish asymptotic normality of this estimate and finally we use this result to obtain the assertion of Theorem 1. For the sake of transparency we assume that $N=n$; the general case is obtained by exactly the same arguments with an additional amount of notation.

For the calculation of the asymptotic bias we first note that it follows from Lemma 2.1 in Dette et al. (2006)

$$
\hat{s}_{I}^{-1}(t)=s^{-1}(t)+\kappa_{2}\left(K_{d}\right) h_{d}^{2}\left(s^{-1}\right)^{\prime \prime}(t)+\Delta_{n}(t)+o\left(h_{d}^{2}\right)+O\left(\frac{1}{n h_{d}}\right),
$$

where the term $\Delta_{n}(t)$ is given by

$$
\Delta_{n}(t)=\frac{1}{n h_{d}} \sum_{i=1}^{n} \int_{-\infty}^{t}\left\{K_{d}\left(\frac{\hat{s}\left(\frac{i}{n}\right)-u}{h_{d}}\right)-K_{d}\left(\frac{s\left(\frac{i}{n}\right)-u}{h_{d}}\right)\right\} \mathrm{d} u=\Delta_{n}^{(1)}(t)+\frac{1}{2} \Delta_{n}^{(2)}(t),
$$


Fig. 5 Left panel: Scatterplot of the nonparametric squared residuals $\hat{\varepsilon}_{i}$. Right panel:

Local-linear estimator for the variance function (dashed line) and its monotonization (solid line)
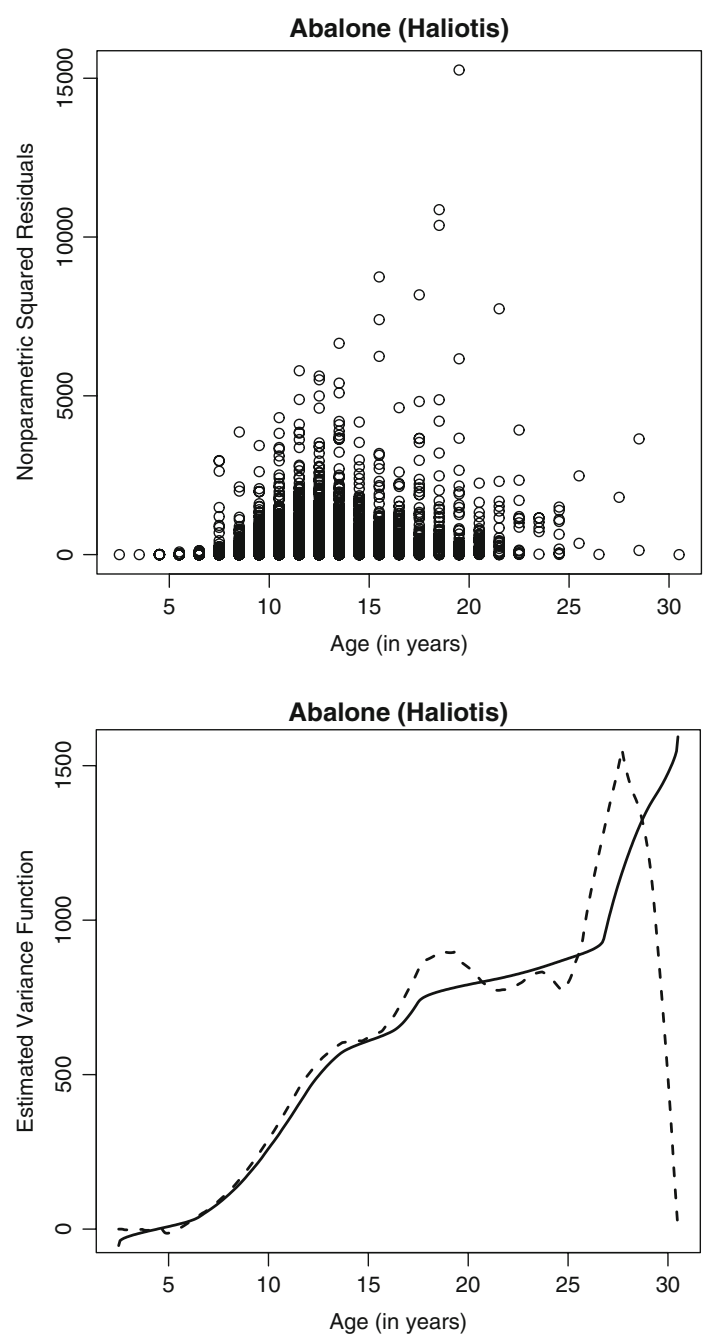

and the quantities $\Delta_{n}^{(j)}(t)(j=1,2)$ in this decomposition are defined by

$$
\begin{aligned}
& \Delta_{n}^{(1)}(t)=\frac{-1}{n h_{d}} \sum_{i=1}^{n} K_{d}\left(\frac{s\left(\frac{i}{n}\right)-t}{h_{d}}\right)\left\{\hat{s}\left(\frac{i}{n}\right)-s\left(\frac{i}{n}\right)\right\} \\
& \Delta_{n}^{(2)}(t)=\frac{1}{n h_{d}^{3}} \sum_{i=1}^{n} \int_{-\infty}^{t} K_{d}^{\prime \prime}\left(\frac{\xi_{i}-u}{h_{d}}\right)\left\{\hat{s}\left(\frac{i}{n}\right)-s\left(\frac{i}{n}\right)\right\}^{2} \mathrm{~d} u
\end{aligned}
$$

with $\left|\xi_{i}-s\left(\frac{i}{n}\right)\right|<\left|\hat{s}\left(\frac{i}{n}\right)-s\left(\frac{i}{n}\right)\right|(i=1, \ldots, n)$. With an appropriate modification at the boundary it follows by similar arguments as in Müller and Stadtmüller (1993) for the second term 


$$
\Delta_{n}^{(2)}(t)=O\left(\frac{1}{h_{d}}\left(h_{r}^{4}+\frac{1}{n h_{r}}\right)\right)
$$

Replacing the density estimate in the denominator of $\hat{s}\left(\frac{i}{n}\right)$ by the positive expressions $n h_{r} f\left(\frac{i}{n}\right)$ we obtain for the first term of the decomposition (42)

$$
\Delta_{n}^{(1)}(t)=\left(\Delta_{n}^{(1.1)}(t)+\Delta_{n}^{(1.2)}(t)+\Delta_{n}^{(1.3)}(t)\right)\left(1+o_{p}(1)\right),
$$

with

$$
\begin{aligned}
& \Delta_{n}^{(1.1)}(t)=\frac{-1}{n^{2} h_{d} h_{r}} \sum_{i, j=1}^{n} K_{d}\left(\frac{s\left(\frac{i}{n}\right)-t}{h_{d}}\right) K_{r}\left(\frac{x_{j}-\frac{i}{n}}{h_{r}}\right) \frac{\left(\Delta_{j}^{\varepsilon}\right)^{2}-s\left(\frac{i}{n}\right)}{f\left(\frac{i}{n}\right)}, \\
& \Delta_{n}^{(1.2)}(t)=\frac{-1}{n^{2} h_{d} h_{r}} \sum_{i, j=1}^{n} K_{d}\left(\frac{s\left(\frac{i}{n}\right)-t}{h_{d}}\right) K_{r}\left(\frac{x_{j}-\frac{i}{n}}{h_{r}}\right) \frac{\left(\Delta_{j}^{m}\right)^{2}}{f\left(\frac{i}{n}\right)}, \\
& \Delta_{n}^{(1.3)}(t)=\frac{-2}{n^{2} h_{d} h_{r}} \sum_{i, j=1}^{n} K_{d}\left(\frac{s\left(\frac{i}{n}\right)-t}{h_{d}}\right) K_{r}\left(\frac{x_{j}-\frac{i}{n}}{h_{r}}\right) \frac{\Delta_{j}^{m} \Delta_{j}^{\varepsilon}}{f\left(\frac{i}{n}\right)}
\end{aligned}
$$

where for $j=1, \ldots, n-r$ the quantities $\Delta_{j}^{\varepsilon}, \Delta_{j}^{m}$ are defined by

$$
\begin{aligned}
\Delta_{j}^{m} & =\sum_{\ell=0}^{r} d_{\ell} m\left(x_{j+\ell}\right) \\
\Delta_{j}^{\varepsilon} & =\sum_{\ell=0}^{r} d_{\ell} \sqrt{s\left(x_{j+\ell}\right)} \varepsilon_{j+\ell},
\end{aligned}
$$

respectively, and we use the notation $\Delta_{j}^{\varepsilon}=\Delta_{j}^{m}=0$, whenever $j \in\{n-r+$ $1, \ldots, n\}$. A straightforward calculation and the assumption of Lipschitz continuity for the regression function show that

$$
\Delta_{j}^{m}=\sum_{\ell=0}^{r} d_{\ell} m\left(x_{j+\ell}\right)=\sum_{\ell=0}^{r-1}\left(\sum_{k=0}^{\ell} d_{k}\right)\left(m\left(x_{j+\ell}\right)-m\left(x_{j+\ell+1}\right)\right)=O\left(\frac{1}{n^{\gamma}}\right)
$$

(uniformly with respect to $j=1, \ldots, n$ ), and it follows that

$$
\Delta_{n}^{(1.2)}(t)=O\left(\frac{1}{n^{2 \gamma}}\right)
$$


Next, consider the first term in (47), which has expectation

$$
\begin{aligned}
E\left[\Delta_{n}^{(1.1)}(t)\right] & =\frac{-1}{n^{2} h_{d} h_{r}} \sum_{i, j} K_{d}\left(\frac{s\left(\frac{i}{n}\right)-t}{h_{d}}\right) K_{r}\left(\frac{x_{j}-\frac{i}{n}}{h_{r}}\right) \frac{\sum_{\ell=0}^{r} d_{\ell}^{2} s\left(x_{j+\ell}\right)-s\left(\frac{i}{n}\right)}{f\left(\frac{i}{n}\right)} \\
& =-\frac{(1+o(1))}{h_{r} h_{d}} \int_{0}^{1} \int_{0}^{1} K_{d}\left(\frac{s(x)-t}{h_{d}}\right) K_{r}\left(\frac{y-x}{h_{r}}\right) f(y) \frac{s(y)-s(x)}{f(x)} \mathrm{d} y \mathrm{~d} x \\
& =-h_{r}^{2} \kappa_{2}\left(K_{r}\right) \int_{0}^{1} \frac{1}{h_{d}} K_{d}\left(\frac{s(x)-t}{h_{d}}\right)\left\{s^{\prime \prime}(x)+\frac{2 s^{\prime}(x) f^{\prime}(x)}{f(x)}\right\} \mathrm{d} x \cdot(1+o(1)) \\
& =-h_{r}^{2} \kappa_{2}\left(K_{r}\right)\left(\frac{s^{\prime \prime} f+2 s^{\prime} f^{\prime}}{f s^{\prime}}\right)\left(s^{-1}(t)\right) \cdot(1+o(1)) .
\end{aligned}
$$

Note that the second equality follows from (2), which yields the approximation

$$
\frac{1}{n}=\int_{x_{j}}^{x_{j+1}} f(u) \mathrm{d} u \approx f\left(x_{j}\right)\left(x_{j+1}-x_{j}\right) .
$$

Note also that we have used the continuity of the function $\left(s^{\prime \prime} f+2 s^{\prime} f^{\prime}\right) /\left(f s^{\prime}\right)$ $\left(s^{-1}(\cdot)\right)$ for the last equality, which follows from the assumptions stated in Sect. 2. With similar calculations the variance of this expression is given by

$$
\operatorname{Var}\left(\Delta_{n}^{(1.1)}(t)\right)=o\left(\frac{1}{n h_{r}}\right)
$$

The remaining third term has obviously expectation $E\left[\Delta_{n}^{(1.3)}(t)\right]=0$, while the second moment can be estimated similarly as in the previous paragraph, that is

$$
\begin{aligned}
E\left[\left(\Delta_{n}^{(1.3)}(t)\right)^{2}\right]= & \frac{4}{n^{4} h_{d}^{2} h_{r}^{2}} \sum_{i, i^{\prime}, j j^{\prime}} K_{d}\left(\frac{s\left(\frac{i}{n}\right)-t}{h_{d}}\right) K_{r}\left(\frac{x_{j}-\frac{i}{n}}{h_{r}}\right) \\
& \times K_{d}\left(\frac{s\left(\frac{i^{\prime}}{n}\right)-t}{h_{d}}\right) K_{r}\left(\frac{x_{j^{\prime}}-\frac{i^{\prime}}{n}}{h_{r}}\right) \frac{\Delta_{j}^{m} \Delta_{j^{\prime}}^{m} E\left[\Delta_{j}^{\varepsilon} \Delta_{j^{\prime}}^{\varepsilon}\right]}{f\left(\frac{i}{n}\right) f\left(\frac{i^{\prime}}{n}\right)} \\
= & O\left(\frac{1}{n^{1+2 \gamma} h_{r}}\right)
\end{aligned}
$$

where we used the fact that $\Delta_{i}^{\varepsilon}$ and $\Delta_{j}^{\varepsilon}$ are uncorrelated, whenever $|i-j|>r$. Therefore Markov's inequality yields

$$
\Delta_{n}^{(1.3)}(t)=O_{p}\left(\frac{1}{n^{1 / 2+\gamma} h_{r}^{1 / 2}}\right)=o_{p}\left(\frac{1}{\sqrt{n h_{r}}}\right)
$$


and a combination with (41), (42), (45), (53), (55) shows that

$$
\begin{aligned}
& \sqrt{n h_{r}}\left\{\hat{s}_{I}^{-1}(t)-s^{-1}(t)-\kappa_{2}\left(K_{d}\right) h_{d}^{2}\left(s^{-1}\right)^{\prime \prime}(t)+h_{r}^{2} \kappa_{2}\left(K_{r}\right)\left(\frac{s^{\prime \prime} f+2 s^{\prime} f^{\prime}}{f s^{\prime}}\right)\left(s^{-1}(t)\right)\right\} \\
& =Z_{n}+o_{p}(1)
\end{aligned}
$$

where the random variable $Z_{n}$ is defined as

$$
Z_{n}=\frac{-1}{n^{3 / 2} h_{d} \sqrt{h_{r}}} \sum_{i, j=1}^{n} K_{d}\left(\frac{s\left(\frac{i}{n}\right)-t}{h_{d}}\right) K_{r}\left(\frac{x_{j}-\frac{i}{n}}{h_{r}}\right) \frac{\left(\Delta_{j}^{\varepsilon}\right)^{2}-E\left[\left(\Delta_{j}^{\varepsilon}\right)^{2}\right]}{f\left(\frac{i}{n}\right)}
$$

For the variance of $Z_{n}$ we obtain

$$
\begin{aligned}
\operatorname{Var}\left(Z_{n}\right)= & \frac{1}{n^{3} h_{d}^{2} h_{r}} \sum_{i, i^{\prime}, j j^{\prime}} K_{d}\left(\frac{s\left(\frac{i}{n}\right)-t}{h_{d}}\right) K_{d}\left(\frac{s\left(\frac{i^{\prime}}{n}\right)-t}{h_{d}}\right) \\
& \times K_{r}\left(\frac{x_{j}-\frac{i}{n}}{h_{r}}\right) K_{r}\left(\frac{x_{j^{\prime}}-\frac{i^{\prime}}{n}}{h_{r}}\right) \frac{L_{j, j^{\prime}}}{f\left(\frac{i}{n}\right) f\left(\frac{i^{\prime}}{n}\right)} \\
= & \frac{(1+o(1))}{n^{3} h_{d}^{2} h_{r}} \sum_{i, i^{\prime}, j} K_{d}\left(\frac{s\left(\frac{i}{n}\right)-t}{h_{d}}\right) K_{d}\left(\frac{s\left(\frac{i^{\prime}}{n}\right)-t}{h_{d}}\right) \\
& \times K_{r}\left(\frac{x_{j}-\frac{i}{n}}{h_{r}}\right) K_{r}\left(\frac{x_{j}-\frac{i^{\prime}}{n}}{h_{r}}\right) \sum_{k=-r}^{r} \frac{L_{j, j+k}}{f\left(\frac{i}{n}\right) f\left(\frac{i^{\prime}}{n}\right)},
\end{aligned}
$$

where the quantities $L_{j, j^{\prime}}$ are defined by

$$
L_{j, j^{\prime}}=E\left[\left(\Delta_{j}^{\varepsilon}\right)^{2}\left(\Delta_{j^{\prime}}^{\varepsilon}\right)^{2}\right]-E\left[\left(\Delta_{j}^{\varepsilon}\right)^{2}\right] E\left[\left(\Delta_{j^{\prime}}^{\varepsilon}\right)^{2}\right]
$$

We now calculate these expectations separately, that is

$$
\begin{aligned}
\sum_{k=-r}^{r} E\left[\left(\Delta_{j}^{\varepsilon}\right)^{2}\right] E\left[\left(\Delta_{j+k}^{\varepsilon}\right)^{2}\right] & =\sum_{k=-r}^{r}\left(\sum_{\ell=0}^{r} d_{\ell}^{2} s\left(x_{j+\ell}\right)\right)\left(\sum_{\ell^{\prime}=0}^{r} d_{\ell^{\prime}}^{2} s\left(x_{j+k+\ell^{\prime}}\right)\right) \\
& =(2 r+1) s^{2}\left(x_{j}\right)(1+o(1))
\end{aligned}
$$

uniformly with respect to $j=1, \ldots, n$, where we used the convention $s\left(x_{i}\right)=0$, whenever $i \notin\{1, \ldots, n\}$. The investigation of the first term in (59) is more difficult, but a straightforward calculation gives 


$$
\begin{aligned}
& \sum_{k=-r}^{r} E\left[\left(\Delta_{j}^{\varepsilon}\right)^{2}\left(\Delta_{j+k}^{\varepsilon}\right)^{2}\right]=\sum_{\ell=0}^{r} d_{\ell}^{4} s^{2}\left(x_{j+\ell}\right) m_{4}\left(x_{j+\ell}\right)+3 \sum_{\substack{\ell, \ell^{\prime}=0 \\
\ell \neq \ell^{\prime}}}^{r} d_{\ell}^{2} d_{\ell^{\prime}}^{2} s\left(x_{j+\ell}\right) s\left(x_{j+\ell^{\prime}}\right) \\
& +2 \sum_{k=1}^{r} \sum_{\ell, \ell^{\prime}, p, p^{\prime}=0}^{r} d_{\ell} d_{\ell^{\prime}} d_{p} d_{p^{\prime}} \sqrt{s\left(x_{j+\ell}\right) s\left(x_{j+\ell^{\prime}}\right) s\left(x_{j+k+p}\right) s\left(x_{j+k+p^{\prime}}\right)} \\
& \times E\left[\varepsilon_{j+\ell} \varepsilon_{j+\ell^{\prime}} \varepsilon_{j+k+p^{\prime}} \varepsilon_{j+k+p}\right] \cdot(1+o(1)) \\
& =s^{2}\left(x_{j}\right)\left\{\left(m_{4}\left(x_{j}\right)-3\right) \sum_{\ell=0}^{r} d_{\ell}^{4}+3+2 m_{4}\left(x_{j}\right) \sum_{k=1}^{r} \sum_{\ell=0}^{r-k} d_{\ell}^{2} d_{\ell+k}^{2}\right. \\
& \left.+2 \sum_{k=1}^{r}\left(\sum_{\substack{\ell, s=0 \\
\ell \neq s+k}}^{r} d_{\ell}^{2} d_{s}^{2}+2 \sum_{\substack{\ell, s=0 \\
\ell \neq s}}^{r-k} d_{\ell} d_{\ell+k} d_{s} d_{s+k}\right)\right\}(1+o(1)) \\
& =s^{2}\left(x_{j}\right)\left\{\left(m_{4}\left(x_{j}\right)-3\right)\left(\sum_{\ell=0}^{r} d_{\ell}^{4}+2 \sum_{k=1}^{r} \sum_{\ell=0}^{r-k} d_{\ell}^{2} d_{\ell+k}^{2}\right)+3\right. \\
& \left.+2 \sum_{k=1}^{r}\left(\left[\sum_{\ell=0}^{r} d_{\ell}^{2}\right]^{2}+2\left[\sum_{\ell=0}^{r-k} d_{\ell} d_{\ell+k}\right]^{2}\right)\right\}(1+o(1)) \\
& =s^{2}\left(x_{j}\right)\left\{m_{4}\left(x_{j}\right)+2 r+4 \sum_{k=1}^{r}\left[\sum_{\ell=0}^{r-k} d_{\ell} d_{\ell+k}\right]^{2}\right\}(1+o(1)) \text {, }
\end{aligned}
$$

uniformly with respect to $j=1, \ldots, n$. Combining (58) - (61) and observing the definition of $\delta_{r}$ in (22) we thus obtain

$$
\begin{aligned}
\operatorname{Var}\left(Z_{n}\right)= & \frac{(1+o(1))}{n^{3} h_{d}^{2} h_{r}} \sum_{i, i^{\prime}, j=1}^{n} K_{d}\left(\frac{s\left(\frac{i}{n}\right)-t}{h_{d}}\right) K_{d}\left(\frac{s\left(\frac{i^{\prime}}{n}\right)-t}{h_{d}}\right) \\
& \times K_{r}\left(\frac{x_{j}-\frac{i}{n}}{h_{r}}\right) K_{r}\left(\frac{x_{j}-\frac{i^{\prime}}{n}}{h_{r}}\right) \frac{\left(m_{4}\left(x_{j}\right)-1+4 \delta_{r}\right) s^{2}\left(x_{j}\right)}{f\left(\frac{i}{n}\right) f\left(\frac{i^{\prime}}{n}\right)} \\
= & \frac{(1+o(1))}{h_{d}^{2} h_{r}} \int_{0}^{1} K_{d}\left(\frac{s(z)-t}{h_{d}}\right) \int_{0}^{1} K_{d}\left(\frac{s(y)-t}{h_{d}}\right) \\
& \times \int_{0}^{1} \frac{s^{2}(x)\left(m_{4}(x)-1+4 \delta_{r}\right)}{f(y) f(z)} K_{r}\left(\frac{x-y}{h_{r}}\right) K_{r}\left(\frac{x-z}{h_{r}}\right) f(x) \mathrm{d} x \mathrm{~d} y \mathrm{~d} z \\
= & \frac{s^{2}\left(s^{-1}(t)\right)\left(m_{4}\left(s^{-1}(t)\right)-1+4 \delta_{r}\right)}{\left(s^{\prime}\left(s^{-1}(t)\right)^{2} f\left(s^{-1}(t)\right)\right.} \iint K_{d}(w) K_{d}(v) K_{r}(u) \\
& \times K_{r}\left(\frac{s^{-1}\left(t+h_{d} v\right)-s^{-1}\left(t+h_{d} w\right)}{h_{r}}+u\right) \mathrm{d} u \mathrm{~d} v \mathrm{~d} w \cdot(1+o(1)) \\
= & \frac{t^{2}\left[m_{4}\left(s^{-1}(t)\right)-1+4 \delta_{r}\right]}{\left(s^{\prime}\left(s^{-1}(t)\right)^{2} f\left(s^{-1}(t)\right)\right.} \int_{-1}^{1} K_{r}^{2}(u) \mathrm{d} u \cdot(1+o(1)) .
\end{aligned}
$$


A similar calculation and an application of Orey's (1958) central limit theorem for arrays of $m$-dependent random variables finally shows that $Z_{n}$ is asymptotically normal distributed, that is

$$
Z_{n} \stackrel{\mathcal{D}}{\longrightarrow} \mathcal{N}\left(0, \xi^{2}(t)\right)
$$

where the asymptotic variance $\xi^{2}(t)$ is defined by

$$
\xi^{2}(t)=\frac{t^{2}\left\{m_{4}\left(s^{-1}(t)\right)-1+4 \delta_{r}\right\}}{\left(s^{\prime}\left(s^{-1}(t)\right)^{2} f\left(s^{-1}(t)\right)\right.} \int_{-1}^{1} K_{r}^{2}(u) \mathrm{d} u,
$$

and from (56) we have

$$
\begin{aligned}
& \sqrt{n h_{r}}\left\{\hat{s}_{I}^{-1}(t)-s^{-1}(t)-\kappa_{2}\left(K_{d}\right) h_{d}^{2}\left(s^{-1}\right)^{\prime \prime}(t)+h_{r}^{2} \kappa_{2}\left(K_{r}\right)\left(\frac{s^{\prime \prime} f+2 s^{\prime} f^{\prime}}{f s^{\prime}}\right)\left(s^{-1}(t)\right)\right\} \\
& \stackrel{\mathcal{D}}{\longrightarrow} \mathcal{N}\left(0, \xi^{2}(t)\right) .
\end{aligned}
$$

The final assertion regarding the asymptotic normality of the estiamte $\hat{s}_{I}$ is now obtained by similar arguments as presented in the proof of Theorem 3.2 in Dette et al. (2006), and for the sake of self-consistency we indicate the main steps in this derivation. For this define for $t \in(0,1)$ the quantity $\varepsilon=$ $\min \{(s(t)-s(0)) / 2,(s(1)-s(t)) / 2\}>0$ and the set $D=(s(t)-\varepsilon, s(t)+\varepsilon)$. It can be shown by similar arguments as in Mack and Silverman (1982) that $\hat{s}$ converges a.s. uniformly to $s$ and consequently the support of $\hat{s}_{I}^{-1}$ (essentially given by $\left.\left[\min _{x \in[0,1]} \hat{s}(x), \max _{x \in[0,1]} \hat{s}(x)\right]\right)$ contains the set $D$ for all sufficiently large $n\left(\geq n_{0}\right)$. We now apply Lemma 1 in the Appendix to the restrictions of the functions $s^{-1}$ and $\hat{s}_{I}^{-1}$ on the set $D$ and obtain

$$
\hat{s}_{I}(t)-s(t)=-\frac{\left(\hat{s}_{I}^{-1}-s^{-1}\right)}{\left(s^{-1}\right)^{\prime}}(s(t))+o_{p}\left(\frac{1}{\sqrt{n h_{r}}}\right),
$$

which yields

$$
\begin{aligned}
& \sqrt{n h_{r}}\left\{\hat{s}_{I}(t)-s(t)-\Gamma\left(h_{d}, h_{r}, t\right)\right\} \\
& =-\sqrt{n h_{r}}\left\{\frac{\left(\hat{s}_{I}^{-1}-s^{-1}\right)}{\left(s^{-1}\right)^{\prime}}(s(t))+\Gamma\left(h_{d}, h_{r}, t\right)\right\}+o_{p}(1) \\
& =-\sqrt{n h_{r}} s^{\prime}(t)\left\{\left(\hat{s}_{I}^{-1}-s^{-1}\right) \circ s(t)\right. \\
& \left.\quad+\kappa_{2}\left(K_{d}\right) \frac{s^{\prime \prime}(t)}{\left(s^{\prime}(t)\right)^{3}} h_{d}^{2}+\kappa_{2}\left(K_{r}\right)\left(\frac{s^{\prime \prime} f+2 s^{\prime} f^{\prime}}{f s^{\prime}}\right)(t) h_{r}^{2}\right\} \\
& \stackrel{\mathcal{D}}{\longrightarrow} \mathcal{N}\left(0,\left(s^{\prime}(t)\right)^{2} \xi^{2}(s(t))\right),
\end{aligned}
$$


where we used (64) and the fact that $s^{\prime \prime} /\left(s^{\prime}\right)^{3}=-\left(s^{-1}\right)^{\prime \prime}$. Finally, a straightforward calculation shows that

$$
\left(s^{\prime}(t)\right)^{2} \xi^{2}(s(t))=\frac{s^{2}(t)\left\{m_{4}(t)-1+4 \delta_{r}\right\}}{f(t)} \int_{-1}^{1} K_{r}^{2}(u) \mathrm{d} u=\beta^{2}(t),
$$

where $\beta^{2}(t)$ is the asymptotic variance defined in (20).

\subsection{Proof of Theorem 2}

The proof of Theorem 2 is performed by similar arguments as the proof of Theorem 1 and for this reason we will only indicate the main differences. First we note that the arguments given at the beginning of the proof of Theorem 1 remain valid. This follows by some standard calculations using the differentiability of the regression function and some basic properties of the Nadaraya-Watson estimate. Therefore we obtain

$$
\begin{aligned}
& \sqrt{n h_{r}}\left\{\hat{s}_{I}^{-1}(t)-s^{-1}(t)-\kappa_{2}\left(K_{d}\right) h_{d}^{2}\left(s^{-1}\right)^{\prime \prime}(t)+h_{r}^{2} \kappa_{2}\left(K_{r}\right)\left(\frac{s^{\prime \prime} f+2 s^{\prime} f^{\prime}}{f s^{\prime}}\right)\left(s^{-1}(t)\right)\right\} \\
& =W_{n}+o_{p}(1)
\end{aligned}
$$

where the statistic $W_{n}$ is defined by

$$
W_{n}=\frac{-1}{n^{3 / 2} h_{d} \sqrt{h_{r}}} \sum_{i, j=1}^{n} K_{d}\left(\frac{s\left(\frac{i}{n}\right)-t}{h_{d}}\right) K_{r}\left(\frac{x_{j}-\frac{i}{n}}{h_{r}}\right) \frac{\tilde{\varepsilon}_{j}^{2}-E\left[\tilde{\varepsilon}_{j}^{2}\right]}{f\left(\frac{i}{n}\right)},
$$

the quantities $\tilde{\varepsilon}_{j}$ are given by

$$
\tilde{\varepsilon}_{j}=\sqrt{s\left(x_{j}\right)} \varepsilon_{j}-\sum_{\ell=1}^{n} w_{j \ell} \sqrt{s\left(x_{\ell}\right)} \varepsilon_{\ell}=\sum_{\ell=1}^{n} w_{j \ell}\left(\sqrt{s\left(x_{j}\right)} \varepsilon_{j}-\sqrt{s\left(x_{\ell}\right)} \varepsilon_{\ell}\right)
$$

and

$$
w_{j \ell}=\frac{K\left(\frac{x_{\ell}-x_{j}}{h}\right)}{\sum_{q=1}^{n} K\left(\frac{x_{q}-x_{j}}{h}\right)}
$$

denote the weights of the Nadaraya-Watson estimate. In the following we will make use of the estimate

$$
W_{n}=V_{n}+o_{p}(1)
$$

where the statistic $V_{n}$ is defined by

$$
V_{n}=\frac{-1}{n^{3 / 2} h_{d} \sqrt{h_{r}}} \sum_{i, j=1}^{n} K_{d}\left(\frac{s\left(\frac{i}{n}\right)-t}{h_{d}}\right) K_{r}\left(\frac{x_{j}-\frac{i}{n}}{h_{r}}\right) \frac{s\left(x_{j}\right)\left(\varepsilon_{j}^{2}-1\right)}{f\left(\frac{i}{n}\right)} .
$$


With this representation it now follows by a similar calculation as given in the proof of Theorem 1 that

$$
\begin{aligned}
\operatorname{Var}\left(V_{n}\right)= & \frac{(1+o(1))}{h_{d}^{2} h_{r}} \iiint K_{d}\left(\frac{s\left(x_{1}\right)-t}{h_{d}}\right) K_{d}\left(\frac{s\left(x_{2}\right)-t}{h_{d}}\right) \\
& \times K_{r}\left(\frac{x_{3}-x_{1}}{h_{r}}\right) K_{r}\left(\frac{x_{3}-x_{2}}{h_{r}}\right) \frac{f\left(x_{3}\right) s^{2}\left(x_{3}\right)\left(m_{4}\left(x_{3}\right)-1\right)}{f\left(x_{1}\right) f\left(x_{2}\right)} \mathrm{d} x_{1} \mathrm{~d} x_{2} \mathrm{~d} x_{3} \\
= & \frac{t^{2}\left(m_{4}\left(s^{-1}(t)\right)-1\right)}{f\left(s^{-1}(t)\right)\left(s^{\prime}\left(s^{-1}(t)\right)^{2}\right.} \int K_{r}^{2}(u) \mathrm{d} u \cdot(1+o(1)),
\end{aligned}
$$

and a straightforward application of Lindeberg's Theorem yields

$$
V_{n} \stackrel{\mathcal{D}}{\Rightarrow} \mathcal{N}\left(0, \tilde{\delta}^{2}(t)\right)
$$

where the asymptotic variance $\tilde{\delta}^{2}(t)$ is defined as

$$
\tilde{\delta}^{2}(t)=\frac{t^{2}\left\{m_{4}\left(s^{-1}(t)\right)-1\right\}}{\left(s^{\prime}\left(s^{-1}(t)\right)^{2} f\left(s^{-1}(t)\right)\right.} \int K_{r}^{2}(u) \mathrm{d} u .
$$

The assertion of Theorem 2 now follows by exactly the same arguments as given at the end of the proof of Theorem 1.

We finally prove the remaining argument for (69) noting that

$$
W_{n}-V_{n}=2 A_{n}-B_{n}
$$

where

$$
\begin{aligned}
A_{n}= & \frac{1}{n^{3 / 2} h_{d} \sqrt{h_{r}}} \sum_{i, j, \ell=1}^{n} K_{d}\left(\frac{s\left(\frac{i}{n}\right)-t}{h_{d}}\right) \\
& \times K_{r}\left(\frac{x_{j}-\frac{i}{n}}{h_{r}}\right) \frac{w_{j \ell} \varepsilon_{j} \varepsilon_{\ell}-E\left[w_{j \ell} \varepsilon_{j} \varepsilon_{\ell}\right]}{f\left(\frac{i}{n}\right)} \sqrt{s\left(x_{j}\right) s\left(x_{\ell}\right)} \\
B_{n}= & \frac{1}{n^{3 / 2} h_{d} \sqrt{h_{r}}} \sum_{i, j, \ell, \ell^{\prime}=1}^{n} K_{d}\left(\frac{s\left(\frac{i}{n}\right)-t}{h_{d}}\right) \\
& \times K_{r}\left(\frac{x_{j}-\frac{i}{n}}{h_{r}}\right) \frac{w_{j \ell} w_{j \ell^{\prime}} \varepsilon_{\ell} \varepsilon_{\ell^{\prime}}-E\left[w_{j \ell} w_{j \ell^{\prime}} \varepsilon_{\ell} \varepsilon_{\ell^{\prime}}\right]}{f\left(\frac{i}{n}\right)} \sqrt{s\left(x_{\ell}\right) s\left(x_{\ell^{\prime}}\right)} .
\end{aligned}
$$


Obviously, we have $E\left[A_{n}\right]=E\left[B_{n}\right]=0$, while we obtain for the variance of $A_{n}$

$$
\begin{aligned}
\operatorname{Var}\left(A_{n}\right)= & E\left[A_{n}^{2}\right] \\
= & \frac{1}{n^{3} h_{d}^{2} h_{r}} \sum_{i, i^{\prime}, j j^{\prime}, \ell, \ell^{\prime}} K_{d}\left(\frac{s\left(\frac{i}{n}\right)-t}{h_{d}}\right) K_{r}\left(\frac{x_{j}-\frac{i}{n}}{h_{r}}\right) \\
& \times K_{d}\left(\frac{s\left(\frac{i^{\prime}}{n}\right)-t}{h_{d}}\right) K_{r}\left(\frac{x_{j^{\prime}}-\frac{i^{\prime}}{n}}{h_{r}}\right) \frac{E\left[w_{j \ell} w_{j^{\prime} \ell^{\prime}} \varepsilon_{j} \varepsilon_{\ell} \varepsilon_{j^{\prime}} \varepsilon_{\ell^{\prime}}\right]}{f\left(\frac{i}{n}\right) f\left(\frac{i^{\prime}}{n}\right)} \sqrt{s\left(x_{j}\right) s\left(x_{j^{\prime}}\right) s\left(x_{\ell}\right) s\left(x_{\ell^{\prime}}\right)} \\
= & \frac{(1+o(1))}{n^{3} h_{d}^{2} h_{r}}\left\{c_{1} \sum_{i, i^{\prime}, j, \ell} K_{d}\left(\frac{s\left(\frac{i}{n}\right)-t}{h_{d}}\right) K_{r}\left(\frac{x_{j}-\frac{i}{n}}{h_{r}}\right) K_{d}\left(\frac{s\left(\frac{i^{\prime}}{n}\right)-t}{h_{d}}\right)\right. \\
& \times K_{r}\left(\frac{x_{j}-\frac{i^{\prime}}{n}}{h_{r}}\right) \frac{w_{j \ell}^{2} s\left(x_{j}\right) s\left(x_{\ell}\right)}{f\left(\frac{i}{n}\right) f\left(\frac{i^{\prime}}{n}\right)} \\
& +c_{2} \sum_{i, i^{\prime}, j, \ell} K_{d}\left(\frac{s\left(\frac{i}{n}\right)-t}{h_{d}}\right) K_{r}\left(\frac{x_{j}-\frac{i}{n}}{h_{r}}\right) K_{d}\left(\frac{s\left(\frac{i^{\prime}}{n}\right)-t}{h_{d}}\right) \\
& \left.\times K_{r}\left(\frac{x_{\ell}-\frac{i^{\prime}}{n}}{h_{r}}\right) \frac{w_{j \ell} w_{\ell j} s\left(x_{j}\right) s\left(x_{\ell}\right)}{f\left(\frac{i}{n}\right) f\left(\frac{i^{\prime}}{n}\right)}\right\}
\end{aligned}
$$

for some constants $c_{1}, c_{2}>0$. Observing the definition of $w_{i j}$ in (68) it therefore follows

$$
\begin{aligned}
\operatorname{Var}\left(A_{n}\right)= & \frac{(1+o(1))}{n h_{d}^{2} h_{r} h^{2}}\left\{c_{1} \iiint \int K_{d}\left(\frac{s\left(x_{1}\right)-t}{h_{d}}\right) K_{r}\left(\frac{x_{2}-x_{1}}{h_{r}}\right)\right. \\
& \times K_{d}\left(\frac{s\left(x_{3}\right)-t}{h_{d}}\right) K_{r}\left(\frac{x_{2}-x_{3}}{h_{r}}\right) \\
& \times K^{2}\left(\frac{x_{2}-x_{4}}{h}\right) \frac{s\left(x_{2}\right) s\left(x_{4}\right) f\left(x_{4}\right) \mathrm{d} x_{1} \mathrm{~d} x_{2} \mathrm{~d} x_{3} \mathrm{~d} x_{4}}{f\left(x_{1}\right) f\left(x_{2}\right) f\left(x_{3}\right)} \\
& +c_{2} \iiint \int K_{d}\left(\frac{s\left(x_{1}\right)-t}{h_{d}}\right) K_{r}\left(\frac{x_{2}-x_{1}}{h_{r}}\right) \\
& \times K_{d}\left(\frac{s\left(x_{3}\right)-t}{h_{d}}\right) K_{r}\left(\frac{x_{4}-x_{3}}{h_{r}}\right) \\
& \left.\times K^{2}\left(\frac{x_{2}-x_{4}}{h}\right) \frac{s\left(x_{2}\right) s\left(x_{4}\right) \mathrm{d} x_{1} \mathrm{~d} x_{2} \mathrm{~d} x_{3} \mathrm{~d} x_{4}}{f\left(x_{1}\right) f\left(x_{3}\right)}\right\} \\
= & O\left(\frac{1}{n h}\right) .
\end{aligned}
$$

A similar but tedious calculation shows that

$$
\operatorname{Var}\left(B_{n}\right)=O\left(\frac{h_{r}}{n h^{2}}\right)
$$


and from (73) the estimate (69) follows, which completes the proof of Theorem 2.

\section{Appendix: An auxilary result}

The following Lemma is required in the proof of Theorem 1. The proof follows by exactly the same arguments as given in the proof of Lemma A.1 in Dette et al. (2006) and is therefore omitted.

Lemma 1 For fixed $t \in \mathbb{R}$ let $\mathcal{M}$ denote the set of all twice continuously differentiable functions of the form $H: D \rightarrow \mathbb{R}$ (for some open set $D \subseteq \mathbb{R}$ ), which contain $t$ in the interior of their image, i.e. $t \in$ int $H(D)$, and have a positive derivative in a neighbourhood of the point $H^{-1}(t)$. For fixed $H_{1}, H_{2} \in \mathcal{M}$ the mapping

$$
Q:\left\{\begin{aligned}
{[0,1] } & \rightarrow \mathbb{R} \\
\lambda & \rightarrow\left(H_{1}+\lambda\left(H_{2}-H_{1}\right)\right)^{-1}(t)
\end{aligned}\right.
$$

is twice continuously differentiable with

$$
\begin{aligned}
Q^{\prime}(\lambda) & =-\frac{\left(H_{2}-H_{1}\right)}{h_{1}+\lambda\left(h_{2}-h_{1}\right)} \circ\left(H_{1}+\lambda\left(H_{2}-H_{1}\right)\right)^{-1}(t) \\
Q^{\prime \prime}(\lambda) & =Q^{\prime}(\lambda)\left\{\frac{-2\left(h_{2}-h_{1}\right)}{h_{1}+\lambda\left(h_{2}-h_{1}\right)}+\frac{\left(H_{2}-H_{1}\right)\left(h_{1}^{\prime}+\lambda\left(h_{2}^{\prime}-h_{1}^{\prime}\right)\right)}{\left\{h_{1}+\lambda\left(h_{2}-h_{1}\right)\right\}^{2}}\right\} \circ Q(\lambda),
\end{aligned}
$$

where $h_{1}, h_{2}$ denote the derivatives of $H_{1}, H_{2}$, respectively.

Acknowledgments The authors are grateful to Isolde Gottschlich who typed numerous versions of this paper with considerable technical expertise and to M. Birke, E. Mammen and W. Polonik for useful discussions and some help with the references. We would also like to thank the referees for their constructive comments on an earlier version of this paper. The work of the authors was supported by the Sonderforschungsbereich 475, Komplexitätsreduktion in multivariaten Datenstrukturen.

\section{References}

Akritas, M., Van Keilegom, I. (2001). Nonparametric estimation of the residual distribution. Scandinavian Journal of Statistics, 28, 549-567.

Box, G. E. P. (1988). Signal to noise ratios, performance criteria and transformation. Technometrics (with discussions), 30, 1-40.

Brunk, H. D. (1955). Maximum likelihood estimates of monotone parameters. The Annals of Mathematical Statistics, 26, 607-616.

Carroll, R. J. (1982). Adapting for heteroscedasticity in linear models. The Annals of Statistics, 10, 1224-1233.

Carroll, R. J. (1987). The effect of variance function estimation on prediction-intervals. In J. O. Berger, S. S. Gupta (eds.), Proceedings of the 4th purdue symposium statistical decision theory and related topics, Vol. II. Heidelberg: Springer.

Dette, H., Munk, A. (1998). Testing heteroscedasticity in nonparametric regression. Journal of the Royal Statistical Society, Series B, 60, 693-708. 
Dette, H., Munk, A., Wagner, T. (1998). Estimating the variance in nonparametric regression - what is a reasonable choice? Journal of the Royal Statistical Society, Series B, 60, 751-764.

Dette, H., Neumeyer, N., Pilz, K. F. (2006). A simple nonparametric estimator of a monotone regression function. Bernoulli, 12, 469-490.

Fan, J., Gijbels, I. (1995). Data driven bandwidth selection in local polynomial fitting: variable bandwidth and spatial adaption. Journal of the Royal Statistical Society, Series B, 57, 371-394.

Fan, J., Gijbels, I. (1996). Local polynomial modelling and its applications. London: Chapman and Hall.

Fan, J., Yao, Q. (1998). Efficient estimation of conditional variance functions in stochastic regression. Biometrika, 85, 645-660.

Gasser, T., Sroka, L., Jennen-Steinmetz, G. (1986). Residual variance and residual pattern in nonlinear regression. Biometrika, 73, 626-633.

Hall, P., Carroll, R. J. (1989). Variance estimation in regression: the effect of estimating the mean. Journal of the Royal Statistical Society, Series B, 51, 3-14.

Hall, P., Huang, L. S. (2001). Nonparametric kernel regression subject to monotonicity constraints. The Annals of Statistics, 29, 624-647.

Hall, P., Kay, J. W., Titterington, D. M. (1990). Asymptotically optimal difference-based estimation of variance in nonparametric regression. Biometrika, 77, 521-528.

Hall, P., Marron, J. S. (1990). On variance estimation in nonparametric regression. Biometrika, 77, 415-419.

Mack, Y. P., Silverman, B. W. (1982). Weak and strong uniform consistency of kernel regression estimates. Zeitschrift Wahrscheinlichkeitstheorie verwandte Gebiete, 61, 405-415.

Mammen, E. (1991). Estimating a smooth monotone regression function. The Annals of Statistics, $19,724-740$.

Mukerjee, R. (1988). Monotone nonparametric regression. The Annals of Statistics, 16, 741-750

Müller, H. G., Stadtmüller, U. (1987). Estimation of heteroscedasticity in regression analysis. The Annals of Statistics, 15, 610-625.

Müller, H. G., Stadtmüller, U. (1993). On variance function estimation with quadratic forms. Journal of Statistical Planning and Inference, 35, 213-231.

Nash, W. J., Sellers, T. L., Talbot, S. R., Cawthorn, A. J., Ford, W. B. (1994). The population biology of abalone (Haliotis) in Tasmania. I. Blacklip abalone (H.rubra) from the north coast and islands of Bass Strait. Sea Fisheries Division, Technical Report No. 48.

Orey, S. (1958). A central limit theorem for $m$-dependent random variables. Duke Mathematical Journal, 25, 543-546.

Rice, J. (1984). Bandwidth choice for nonparametric regression. The Annals of Statistics, 12, $1215-1230$.

Ruppert, D., Wand, M. P., Holst, U., Hössjer, O. (1997). Local polynomial variance-function estimation. Technometrics, 39, 262-273.

Sacks, J., Ylvisaker, D. (1970). Designs for regression problems for correlated errors. The Annals of Mathematical Statistics, 41, 2057-2074.

Yu, K., Jones, M. C. (2004). Likelihood-based local linear estimation of the conditional variance function. Journal of the American Statistical Association, 99, 139-155. 